\title{
Optimal Design and Operational Planning of Responsive Process Supply Chains
}

\author{
Fengqi You and Ignacio E. Grossmann \\ Center for Advanced Process Decision-making \\ Department of Chemical Engineering \\ Carnegie Mellon University \\ Pittsburgh, Pennsylvania 15213-3890, USA
}

\section{ABSTRACT}

This chapter addresses the problem of optimal design and operational planning of multi-echelon, multi-site process supply chain networks (PSCNs) with consideration of responsiveness and profitability. A quantitative characterization of responsiveness for PSCNs is presented, which measures the response time or lead time to changes in demands assuming zero inventories. This measure is incorporated in a multi-period mixed-integer non-linear programming (MINLP) model, which considers the selections of suppliers and manufacturing sites, process technology, production levels and scheduling. The problem is formulated as a bi-criterion optimization model in which the objectives are to maximize the net present value (NPV) and to minimize the lead time. This allows establishing trade-offs between the economics and responsiveness of the supply chain network. The model produces a Pareto-optimal curve, which reveals how the optimal net present value, and therefore the network structure of the PSCN, changes with different specifications of lead time. The application of this model is illustrated through an example. The modeling approach developed in this paper and the results obtained suggest that a new conceptual strategy has been developed that yields useful insights regarding the responsiveness of process supply chain networks.

Keywords: Supply Chain Management, Responsiveness, Lead Time, MINLP, Multiobjective Optimization 


\section{INTRODUCTION}

The importance of response time as a competitive advantage for customer satisfaction and market understanding has been recognized for some time (Stalk, 1988). There is a growing recognition that individual business no longer compete as stand-alone entities, but rather as supply chains (Christopher and Towill, 2001), whose success or failure is ultimately determined in the marketplace by the end consumer. The need to meet the demands of customers for ever-shorter lead times, and to synchronize the supply to meet the peaks and troughs of demand, requires the capability to manage the supply chain in a way that enables quick response (Sabath, 1998), which is of critical importance in this area of time-based competition. Responsiveness is defined as the ability of a supply chain to respond rapidly to changes in demand, both in terms of volume and mix of products (Christopher, 2000). In today’s rapid changing industrial environment, responsiveness has become not only the linchpin of companies to competitive success but also the key to survival (Fisher, 1997; Christopher, 2000, 2005).

In the process industries, Enterprise-wide Optimization (EWO) has become a major goal due to the increasing pressure for remaining competitive in the global marketplace (Grossmann, 2005). EWO involves optimizing the operations of supply, manufacturing and distribution activities of a company to reduce costs. While there has been considerable progress in the development of the planning and scheduling models that can be used to address specific problems, a major challenge that remains is to integrate the consideration of responsiveness into the design and operations of supply chain networks. 
However, most of the research on responsive supply chains has been approached qualitatively and no mathematical models are available for its modeling and optimization. The major goal of this chapter is to propose a novel design and operational planning optimization model of PSCNs that takes into account the responsiveness. In this work, we propose a quantitative characterization of responsiveness for process supply chain networks (PSCNs), which determines the lead time under the assumption of zero inventories. We incorporate this measure into a multi-period mixed-integer non-linear programming model, which takes into account the selections of suppliers, manufacturing sites, process technology, production levels and cyclic scheduling. Multi-period modeling allows economic parameters, such as demand and price, to vary with time. To establish the trade-offs between the economics and responsiveness of the supply chain network, we formulate a bi-criterion optimization model in which the objectives are to maximize the net present value (NPV) and to minimize the lead time. The multi-objective optimization model yields a Pareto-optimal curve, which reveals how the optimal net present value, and therefore the network structure of the PSCN, changes with different specifications of responsiveness.

The paper is organized as follow. We first review the relevant literature. This is followed by a motivating example and a formal definition of the problem addressed in this paper. Next, the MINLP formulation for the multi-objective optimization model is described. Results for an example are presented and conclusions are drawn about this work. 


\section{LITERATURE REVIEW}

The "responsiveness" literature for supply chains is highly qualitative and conceptual, and has not been subjected to the kind of quantitative analysis that is proposed in this chapter. There are, however, related works that offer relevant insights. Forrester (1961) illustrated in a series of case studies the effect of dynamics in industrial systems, which gives rise to the "bullwhip effect”. Lee et al (1997) further demonstrated that "bullwhip" effect is a consequence of the information delay due to the structure of supply chains, and the severity of this effect is positively related to lead times. Responsiveness in the wider supply chain context has been discussed by Fisher (1997), who argues that the product characteristics (innovative or functional) and life cycles need to be linked to the layout and functions (conversion and market mediation) of the supply chain. He also pointed out that need reducing the lead time enable quick response to unpredictable demand to minimize stockouts, markdowns and obsolete inventory. Matson et al (1999) discussed the concepts and issues associated with responsiveness in production and illustrate the audit tools they proposed from a case study in the steel industry. Recently, several conceptual models on supply chain responsiveness have been proposed. Christopher and Towill (2001) integrate lead time and agility to highlight the differences in their approach, and combined them to propose an integrated hybrid strategy for designing cost-effective responsive supply chain with seamless connection between manufacturing and logistics. In a later work, Yusuf et al (2004) have reviewed emerging patterns for creating responsive supply chain based on survey research driven by a conceptual model. Holweg (2005) proposed in his paper that product, process and volume are three key factors that determine the responsiveness of a supply chain system, and provided guidelines on how to align the supply chain strategy to these three factors in 
order to balance responsiveness to customer demand and supply chain efficiency. An examination on supply chain system in process industries from a responsiveness view point was carried out by Shaw et al (2005). These authors also proposed a conceptual management strategy to improve the responsiveness of process supply chain system.

Another group of relevant papers to be considered are on supply chain design and operation. A general review of this area is given in Kok and Graves (2003), and a specific review for supply chains in process industries is presented by Shah (2005). Some recent works include the following. Tsiakis et al (2001) presented a supply chain design model for the steady-state continuous processes. Their supply chain model was developed based on determining the connection between multiple markets and multiple plants with fixed locations. Jackson and Grossmann (2003) presented a temporal decomposition scheme based on Lagrangean decomposition for a nonlinear programming problem that models multi-site production planning and distribution models, where nonlinear terms arises from the relationship between production and physical properties or blending ratios. Schulz et al (2005) described two multi-period MINLP models for short term planning of petrochemical complexes. Linearization techniques are applied to reformulate the nonconvex bilinear constraints as MILP models. Recently, Sousa et al (2006) presented a two stage procedure for supply chain design with responsiveness testing. In the first stage, they design the supply chain network and optimize the production and distribution planning over a long time horizon. In the second stage, responsiveness of the first stage decisions are assessed using the service level to the customers (i.e. delay in the order fulfillment). However, all these models consider supply chain networks with only dedicated processes. Multiproduct batch plants or flexible processes were not taken into account, and hence no scheduling models were included. 
There are works on supply chain optimization with consideration of flexible processes in the production network, but most of them are restricted to planning and scheduling for a given facility in a fixed location, without extension to the multisite supply chain network design problems. Bok et al (2000) proposed a multiperiod supply chain optimization model for operational planning of continuous flexible process networks, where sales, intermittent deliveries, production shortfalls, delivery delays, inventory profiles and job changeovers are taken into account. A bilevel decomposition algorithm was proposed, which proved to reduce the computational time significantly. Kallrath (2002) describes a tool for simultaneous strategic and operational planning in a multi-site production network, where key decisions include operating modes of equipment in each time period, production and supply of products, minor changes to the infrastructure and raw material purchases and contracts. A multiperiod model is formulated where equipment may undergo one mode change per period. The standard material balance equations are adjusted to account for the fact that transportation times are much shorter than the period durations. Chen et al (2003) presented a multi-product, multistage and multiperiod production and distribution planning model. They also proposed a two-phase fuzzy decision making method to obtain a compromise solution among all participants of the multi-enterprise supply chain.

Thus, these papers either focus only on the long-term strategic design models, or else are restricted to short-term planning and scheduling models. Hence, no quantitative analyses are available for responsive supply chains. It is the goal of this paper to integrate supply chain decisions in different time scales (design, planning and scheduling), taking into account responsiveness with a quantitative approach. A multi- 
objective optimization procedure is used in this work for trading off the two different types of objectives - economics and responsiveness.

\section{MOTIVATING EXAMPLE}

To illustrate the issues associated with the problems addressed in this paper, consider the example in Figure 1, which is taken from a real world application. A company wants to design its supply chain network in the United States for producing polystyrene resins. Three types of candidate plants are included in the process network (Figure 1). Plant $I$ is used to produce styrene monomers from ethylene and benzene; Plant $I I$ is a multiproduct plant for the production of three different types of solid polystyrene (SPS) resins; Plant III is also a multiproduct plant for the production of two different types of expandable polystyrene (EPS) resins. As shown in Figure 2, two potential ethylene suppliers are located in $\mathrm{OH}$ and $\mathrm{TX}$, and two potential benzene suppliers are located in VA and TX. Three potential manufacturing sites can be located in PA, TX and AL. The PA manufacturing site can set up all the three types of plants, the TX manufacturing site can only install Plant $I$, and the AL manufacturing site can only set up Plants $I I$ and $I I I$. The customers for SPS resins are located in CA and NY, while customers for EPS resins are located in GA and MN. The corresponding superstructure of the supply chain network is given in Figure 3. Based on the given information, we want to design an economic and responsive supply chain by making decisions on design (e.g. selection of suppliers, plant sites and transportation link) and operations (e.g. amount of purchase, sale, production and transportation). This problem is stated in general form in the next section. 


\section{PROBLEM STATEMENT}

In general terms, the problem addressed in this work can be stated as follows. Given is a potential process supply chain network that includes possible manufacturing sites, suppliers and customers (as shown in Figure 4). A set of processes and a time horizon consisting of a number of time periods are given. The processes may be dedicated or flexible. Flexible processes are multi-product processes that operate under different production schemes, using different raw materials and/or producing different products. The PSCN also involves a set of chemicals, which includes raw materials, intermediate products and final products. Demands are assumed to be known in each time period. Raw materials are subject to availability constraints and final products are subject to demand constraints (i.e., within lower and upper bounds). Prices for raw materials and final products are assumed to be known over the entire time horizon. For all production schemes, mass balances are expressed linearly in terms of the main product's production. The investment costs for installing the plants are expressed by a cost function with fixed charges (Figure 5). There could be different transportation types, continuous (e.g. pipelines) or discrete (e.g. barges, rail cars, tanker), for each route which connects the suppliers, plant sites and customers. For simplicity, we will assume that all the transportations of materials in this problem are continuous. Thus, fixed charge cost functions provide good estimations of transportation costs, and inventories for single product plants can be neglected. The transportation times of each route and the residence times of each product are assumed to be known.

As discussed before, responsiveness is the ability of supply chains to respond to the change of customer demands and preferences (Holweg, 2005). Lead time is the time of a supply chain network to respond to customer demand and preference changes in the worst case. Therefore, by assuming zero inventories, lead time will be used as a 
measure of responsiveness (Lee et al, 1997; Yusuf et al, 2004). As shown in Figure 6, a supply chain network with long lead time implies that its responsiveness is low, and vice versa. Thus, to design a responsive supply chain, one objective function of this problem is to minimize the lead time of the entire supply chain network. From the economic aspect, another objective function is to maximize the net present value (NPV) over the specified long-range time horizon. The income from sales, along with investment, operating, transportation and purchase costs are taken into account in the NPV objective function.

Since the two conflicting objectives need to be optimized simultaneously, the corresponding problem yields an infinite set of alternative solutions denoted by the Pareto-optimal curve. These solutions are Pareto-optimal, in the sense that it is impossible to improve both objective functions simultaneously (Halsall and Thibault, 2006). This implies that any designs, for which the net present value and the lead time can be improved simultaneously, are "inferior" solutions that do not belong to the Pareto-optimal curve. The aim of this problem is to determine the supply chain network configurations that define the Pareto optimal solution by maximizing NPV and minimizing the lead time.

\section{MODEL}

The model will be formulated as a multi-period MINLP problem, which predicts the detailed design decisions, production profiles and schedules for the PSCN with different specifications of the lead time. A list of indices, sets, parameters and variables are given in the Appendix. Three types of constraints are included in this model. They are network structure constraints, operational planning constraints and cyclic scheduling constraints. 
Constraints (1) to (8) determine the network structure, constraints (9) to (14) refer to the operational planning constraints, constraints (15) to (30) are used for the cyclic scheduling of multi-product plants. Finally, inequalities (31) to (33) define the lead time and equation (35) defines the net present value, both of which are objective functions to be optimized.

\subsection{Network Structure Constraints}

To determine the topology of network structure and model the selection of suppliers, plant sites, together with the transportation links between them, the binary variables $\left(Y_{k, i}^{P}, Y_{k, l}^{O}, Y_{k, k^{\prime}}^{I}\right)$ for plants and transportation links are introduced for design decisions. Three types of network structure constraints are applied to represent the relationships between each node in the supply chain network.

\subsubsection{Supplier - Plant Site}

The first type of relationship is between suppliers and plant sites, as shown in Figure 7. A transportation link for raw material $j$ from supplier $l s$ to plant site $k$ exists, only if at least one plant that consumes raw material $j$ exists in site $k$. The relationships discussed above can be expressed by the following logic proposition:

$$
Y_{k, l s}^{O} \Rightarrow \vee_{i \in I_{j}} Y_{k, i}^{P}
$$

These logic propositions can be further transformed into inequalities as described in Raman and Grossmann (1993).

$$
Y_{k, l s}^{O} \leq \sum_{i \in I_{j}} Y_{k, i}^{P} \quad \forall k \in K_{i}, l s \in L S_{j}
$$

On the plant side, if a plant that consumes raw material $j$ is set up, at least one transportation link from the supplier $l s$ to site $k$ must be selected. The logic propositions are: 


$$
Y_{k, i}^{P} \Rightarrow \vee_{l s \in L S_{j}} Y_{k, l s}^{O}
$$

Then they can be transformed to inequalities:

$$
Y_{k, i}^{P} \leq \sum_{l s \in L S_{j}} Y_{k, l s}^{O} \quad \forall k \in K_{i}, i \in I_{j}
$$

\subsubsection{Plant site - Customer}

The second type of relationship is between plant sites and customers as shown in Figure 8. A transportation link for product $j$ from plant site $k$ to customer $l d$ exists, only if at least one plant that consumes raw material $j$ exists in site $k$. On the plant side, if a plant that consumes raw material $j$ is set up, there should be at least one link from the customer $l d$ to site $k$ exists. Similarly by transforming from the corresponding logic propositions, leads to the following inequalities:

$$
\begin{array}{ll}
Y_{k, l d}^{O} \leq \sum_{i \in O_{j}} Y_{k, i}^{P} & \forall k \in K_{i}, l d \in L D_{j}, j \in J P_{k, i} \\
Y_{k, i}^{P} \leq \sum_{l d \in L D_{j}} Y_{k, l d}^{O} & \forall k \in K_{i}, i \in O_{j}, j \in J P_{k, i}
\end{array}
$$

\subsubsection{Input and Output of a Plant}

The third type of network structure relationship is the input and output relationship of a plant as in Figure 9. This type of relationship is somewhat more complicated than the previous two, because the inter-site transportation must be taken into account. If an inter-site transportation link from site $k$ to site $k^{\prime}$ is installed for chemical $j$, it implies that at least one plant $i$ in site $k$ is installed that produces chemical $j$, and also at least one plant $i^{\prime}$ in site $k^{\prime}$ is installed that consumes chemical $j$,

$$
\begin{array}{ll}
Y_{k^{\prime}, k}^{I} \leq \sum_{i \in I_{j}} Y_{k, i}^{P} & \forall k, k^{\prime} \in K, j \in\left(J M_{k, i} \cap J P_{k^{\prime}, i^{\prime}}\right) \\
Y_{k, k^{\prime}}^{I} \leq \sum_{i \in O_{j}} Y_{k, i}^{P} & \forall k, k^{\prime} \in K, j \in\left(J P_{k, i} \cap J M_{k^{\prime}, i^{\prime}}\right)
\end{array}
$$


If a plant $i$ in site $k$ is installed, that consumes chemical $j$, then site $k$ is connected to one of the suppliers of chemical $j$ denoted as $l s$, or connected to another site $k^{\prime}$ that produces chemical $j$, or there is another plant $i^{\prime}$ in site $k$ that produces chemical $j$. The logic propositions can be written as follow:

$$
Y_{k, i}^{P} \Rightarrow \vee_{l s \in L S_{j}} Y_{k, l s}^{O} \vee_{k^{\prime} \in K_{i^{\prime}}} Y_{k^{\prime}, k}^{I} \vee_{i^{\prime} \in O_{j}} Y_{k, i^{\prime}}^{P}
$$

which can be transformed into inequalities as:

$$
Y_{k, i}^{P} \leq \sum_{l s \in L S_{j}} Y_{k, l s}^{O}+\sum_{k^{\prime} \in K_{i^{\prime}}} Y_{k^{\prime}, k}^{I}+\sum_{i^{\prime} \in O_{j}} Y_{k, i^{\prime}}^{P} \quad \forall k \in K_{i}, i \in I_{j}, j \in J M_{k, i}
$$

Similarly, if the chemical $j$ is produced by plant $i$ in site $k$, then at least one other plant $i^{\prime}$ in the same site is installed that consumes chemical $j$, or there is at least one transportation link to a customer or the other site that consumes chemical $j$ :

$$
Y_{k, i}^{P} \leq \sum_{l d \in L D_{j}} Y_{k, l d}^{O}+\sum_{k^{\prime} \in K_{i^{\prime}}} Y_{k, k^{\prime}}^{I}+\sum_{i^{\prime} \in I_{j}} Y_{k, i^{\prime}}^{P} \quad \forall k \in K_{i}, i \in O_{j}, j \in J P_{k, i}
$$

Constraints (7) and (8) are defined for all the chemicals (raw materials, intermediate products, final products). When the chemical $j$ is raw material, constraint (7) will reduce to constraint (2); when the chemical $j$ is final product, constraint (8) will reduce to constraint (4).

\subsection{Operational Planning Constraints}

In the operational planning model, investment in plant capacity, and purchase, sale, production, transportation and mass balance relationships are considered, together with the restriction of these activities due to the supply chain structure.

\subsubsection{Production Constraints}

All the chemical flows $W_{k, i, j, s, t}$ associated with production scheme $s$ in plant $i$ of site $k$ other than the main product $j$ are given by the mass balance coefficient. The 
following equation relates the input $\left(W_{k, i, j, s, t}\right)$ to the output $\left(W_{k, i, j}{ }^{\prime}, s, t\right)$ of each process by the material balance coefficient $\mu_{i, j, s}$ :

$$
W_{k, i, j, s, t}=\mu_{i, j, s} W_{k, i, j}, s, t \quad \forall k \in K_{i}, i \in I_{j}, j \in J_{i^{\prime}, s}, j^{\prime} \in J P_{i, s}, s \in S_{i}, t \in T
$$

The production amount should not exceed the design capacity $Q_{k, i}$ for each process:

$$
W_{k, i, j, s, t} \leq \rho_{i, s} Q_{k, i} \text { Lenp }_{t} \quad \forall k \in K_{i}, i \in O_{j}, j \in J P_{k, i}, s \in S_{i}, t \in T
$$

For flexible processes, the maximum production rate $r_{k, i, s}$ of the each main product $j$ of production scheme $s$ is proportional to the capacity of the plant (see Norton and Grossmann, 1994):

$$
r_{k, i, s}=\rho_{i, s} Q_{k, i} \quad \forall k \in K_{i}, i \in O_{j}, j \in J P_{k, i}, s \in S_{i}
$$

\subsubsection{Sale Constraints}

The amount of sale $S_{k, j, l d, t}$ has to lie between given lower bounds $d_{j, l d, t}^{L}$ and upper bounds $d_{j, d, t}^{U}$ of the demand:

$$
d_{j, l d, t}^{L} \leq \sum_{k \in K} S_{k, j, l d, t} \leq d_{j, l d, t}^{U} \quad \forall j \in J, l d \in L D, t \in T
$$

\subsubsection{Upper Bound Constraints}

Purchases $P_{k, j, l s, t}$ from supplier $l s$ to plant site $k$ take place only if the transportation link between them is set up:

$$
P_{k, j, l s, t} \leq P_{k, j, s, t}^{U} Y_{k, l s}^{I} \quad \forall k, j, l s, t
$$

Inter-site transportation $T R_{k, k^{\prime}, j, t}$ from site $k$ to site $k^{\prime}$ take place only if the transportation link between them is set up:

$$
T R_{k, k^{\prime}, j, t} \leq T R_{k, k^{\prime}, j, t}^{U} Y_{k, k^{\prime}}^{I} \quad \forall k, k^{\prime}, j, t
$$

Sales $S_{k, j, l d, t}$ from plant site $k$ to customer $l d$ take place only if the transportation link between them is selected: 


$$
S_{k, j, l d, t} \leq S_{k, j, l d, t}^{U} Y_{k, l d}^{O} \quad \forall k, j, l d, t
$$

Nonzero production flows $W_{k, i, j, s, t}$ are allowed in plant $i$ of site $k$ only if the plant is installed:

$$
\begin{array}{ll}
W_{k, i, j, s, t} \leq W_{k, i, j, s, t}^{U} Y_{k, i}^{P} & \forall k, i, j, s, t \\
Q_{k, i} \leq Q_{k, i}^{U} Y_{k, i}^{P} & \forall k, i
\end{array}
$$

\subsubsection{Mass Balance Constraints}

Mass balance for chemical $j$ in manufacturing site $k$ at time period $t$ is given as follows:

$$
\begin{gathered}
\sum_{l \in \in S} P_{k, j, l s, t}+\sum_{k \in K} T R_{k, k^{\prime}, j, t}+\sum_{i \in O_{j}} \sum_{s \in S_{i}} W_{k, i, j, s, t}=\sum_{l d \in L D} S_{k, j, l d, t}+\sum_{k^{\prime} \in K} T R_{k, k^{\prime}, j, t}+\sum_{i^{\prime} \in I_{j}} \sum_{S^{\prime} \in S_{i}} W_{k, i^{\prime}, j, s^{\prime}, t} \\
\forall k \in K_{i}, j \in J, t \in T
\end{gathered}
$$

\subsection{Cyclic Scheduling Constraints}

To address detailed operations of the multi-product plants, we have considered a cyclic scheduling policy (Pinto and Grossmann, 1994). Under this policy, the sequences to produce each product are decided, together with the cycle time (Figure 10, 11), and then identical schedule is repeated over each time period (Figure 12). The trade-offs between inventories and transitions are established by optimizing the cycle times (as shown in Figure 13).

Important decisions in cyclic scheduling including the sequence of production $\left(Y_{k, i, s, s l, t}^{S}\right)$ and precedence relationship for changeovers between pairs of products $\left(Z_{k, i, s, s^{\prime}, s l, t}\right)$, are restricted by assignment and sequence constraints (constraints (15) to (19)). 


\subsubsection{Assignment Constraints}

The assignment constraints state that exactly one time slot must be assigned to one production scheme and vice versa. The total number of time slots will be exactly equal to the total number of products.

$$
\begin{array}{ll}
\sum_{s l \in S L_{i}} Y_{k, i, s, s l, t}^{S}=1 & \forall k \in K_{i}, i \in I_{j}, s \in S_{i}, t \in T \\
\sum_{s \in S_{i}} Y_{k, i, s, s l, t}^{S}=1 & \forall k \in K_{i}, i \in I_{j}, s l \in S L_{i}, t \in T
\end{array}
$$

\subsubsection{Sequence Constraints}

The sequence constraints state that exactly one transition from product $s$ occurs in the beginning of any time slot if and only if $s$ was being processing during the previous time slot. On the other hand, exactly one transition to product $s$ occurs in the time slot if and only if product $s$ is being processed during that time slot. As suggested in Wolsey (1997), the transition variables $Z_{k, i, s, s^{\prime}, s, t}$ can be replaced by continuous variables between 0 and 1 , instead of binary variables. This significantly reduces the number of discrete variables and improves the computational efficiency.

$$
\begin{array}{ll}
\sum_{s \in S_{i}} Z_{k, i, s, s^{\prime}, s l, t}=Y_{k, i, s^{\prime}, s l-1, t}^{S} & \forall k \in K_{i}, i \in I_{j}, s^{\prime} \in S_{i}, s l \in S L_{i}, t \in T \\
\sum_{s^{\prime} \in S_{i}^{\prime}} Z_{k, i, s, s, s l, t}=Y_{k, i, s, s l, t}^{S} & \forall k \in K_{i}, i \in I_{j}, s \in S_{i}, s l \in S L_{i}, t \in T \\
0 \leq Z_{k, i, s, s^{\prime}, s l, t} \leq 1 & \forall k, i, s, s^{\prime}, s l, t
\end{array}
$$

\subsubsection{Production Constraints}

The production amount of product $s$ in a cycle $\left(W_{k, i, s, t}^{S}\right)$ is equal to the processing rate $r_{k, i, s}$ times the processing time $\delta_{k, i, s, s l, t}$ :

$$
W_{k, i, s, t}^{S}=\sum_{s l \in S L_{i}} r_{k, i, s} \delta_{k, i, s, s l, t} \quad \forall k \in K_{i}, i \in I_{j}, s \in S_{i}, t \in T
$$


The amount to produce for each product in time period $t$ ( $N_{k, i, t}$ cycles in the time period) should be no less than the total production predicted from operational planning in this time period:

$$
W_{k, i, s, t}^{S} N_{k, i, t} \geq W_{k, i, j, s, t} \quad \forall k \in K_{i}, i \in O_{j}, j \in J P_{k, i}, s \in S_{i}, t \in T
$$

\subsubsection{Timing Constraints}

Constraints (22) to (28) are used to restrict the timing issues in the cyclic scheduling.

The processing time $\delta_{k, i, s, s l, t}$ in a certain time slot is equal to the summation of the processing times assigned to all the products in this time slot

$$
\delta_{k, i, s l, t}=\sum_{s \in S_{i}} \delta_{k, i, s, s l, t} \quad \forall k \in K_{i}, i \in I_{j}, s l \in S L_{i}, t \in T
$$

The cycle time $T C_{k, i, t}$ is equal to the summation of all the processing times in each time slot plus the summation of transition times in this cycle

$$
T C_{k, i, t}=\sum_{s l \in S L_{i}} \delta_{k, i, s l, t}+\sum_{s \in S_{i}} \sum_{s^{\prime} \in S_{i}} \sum_{s l \in S L_{i}} Z_{k, i, s, s^{\prime}, s l+1, t} \tau_{i, s, s^{\prime}} \quad \forall k \in K_{i}, i \in I_{j}, t \in T
$$

The start time $T s_{k, i, s l, t}$ and end time $T e_{k, i, s l, t}$ of each time slot are given by:

$$
\begin{array}{ll}
\delta_{k, i, s l, t}=T e_{k, i, s l, t}-T s_{k, i, s l, t} & \forall k \in K_{i}, i \in I_{j}, s l \in S L_{i}, t \in T \\
T s_{k, i, 1, t}=\sum_{s \in S_{i}} \sum_{s^{\prime} \in S_{i}} Z_{k, i, s, s^{\prime}, 1, t} \tau_{i, s, s^{\prime}} & \forall k \in K_{i}, i \in I_{j}, t \in T \\
T s_{k, i, s l, t}=T e_{k, i, s l-1, t}+\sum_{s \in S_{i}} \sum_{s^{\prime} \in S_{i}} Z_{k, i, s, s^{\prime}, s l, t} \tau_{i, s, s^{\prime}} & \forall k \in K_{i}, i \in I_{j}, s l \in S L_{i}, t \in T
\end{array}
$$

The total production time should not exceed the duration of each time period $H_{k, i, t}$ :

$$
T C_{k, i, t} N_{k, i, t} \leq H_{k, i, t} \quad \forall k \in K_{i}, i \in I_{j}, t \in T
$$

The production for scheme $s$ in time slot $s l$ can take place only if the time slot is assigned to the production scheme:

$$
\delta_{k, i, s, s l, t} \leq \delta_{k, i, s, s l, t}^{U} Y_{k, i, s, s l, t}^{S} \quad \forall k \in K_{i}, i \in I_{j}, s \in S_{i}, s l \in S L_{i}, t \in T
$$




\subsubsection{Cost Constraints}

To integrate the cyclic scheduling with the strategic planning, the inventory and transition costs from cyclic scheduling are considered as part of the operating cost. Constraint (29) represents that cost from scheduling in a time period for a certain plant. The first term on the right hand side of the equation stands for the total transition cost in a time period. The second term on the right hand side of the equation is the inventory cost for all the chemicals involved in the production. The change of inventory level in a time period is given in Figure 14. In the work by Pinto and Grossmann (1994), they consider inventory only for final products, as their model is for single plant. In our case, each manufacturing site may have more than one production plant, and inventory for materials of multi-product plants must be also taken into account. Since we assume that material balances are expressed linearly in terms of the main product's production, the cumulative inventory levels for raw materials are also related linearly to the cumulative inventory level of main product in each production scheme and the coefficients of the linear relationships are exactly the absolute value of mass balance coefficients. This leads to the second term on the right hand side of the following constraint. Thus, operating cost for the inventory and transition of flexible processes is given by:

$$
\begin{gathered}
\operatorname{COST}_{k, i, t}^{S}=\sum_{s \in S_{i}} \sum_{s^{\prime} \in S_{i}} \sum_{s l \in S L_{i}} C T R_{i, s, s^{\prime}} Z_{k, i, s, s, s l, t} N_{k, i, t}+\sum_{s \in S_{i}} \sum_{j \in J_{i}} \sum_{s l \in S L_{i}}\left(\left|\mu_{i, j, s}\right| \varepsilon_{j}\right)\left(r_{k, i, s} H_{k, i, t}-W_{k, i, s, t}^{S} N_{k, i, t}\right) \delta_{k, i, s l, t} / 2 \\
\forall k \in K_{i}, i \in I_{j}, t \in T
\end{gathered}
$$

This constraint is nonlinear and nonconvex, with bilinear and triple-linear term. If all the processes in the production network are dedicated, cyclic scheduling need not be taken into account and thus this constraint can be discarded. 


\subsubsection{Upper Bound Constraints}

As a multi-site problem, we need to make sure that if a plant $i$ in site $k$ is not installed, the associated scheduling cost should be 0 . To model this, we introduce the upper bound constraint (30) for the number of cycles $N_{k, i, t}$ in each time period for each multiproduct plant in each manufacturing site:

$$
N_{k, i, t} \leq N_{k, i, t}^{U} Y_{k, i}^{P} \quad \forall k \in K_{i}, i \in I_{j}, t \in T
$$

Also assignment constraints are revised, so that all the scheduling activities can take place only if the plant is installed:

$$
\begin{array}{ll}
\sum_{s l \in S L_{i}} Y_{k, i, s, s l, t}^{S}=Y_{k, i}^{P} & \forall k \in K_{i}, i \in I_{j}, s \in S_{i}, t \in T \\
\sum_{s \in S_{i}} Y_{k, i, s, s l, t}^{S}=Y_{k, i}^{P} & \forall k \in K_{i}, i \in I_{j}, s l \in S L_{i}, t \in T
\end{array}
$$

\subsection{Lead Time Definition}

One of the challenges in this work is to quantitatively define the lead time of PSCNs, which is a quantitative measure of responsiveness. The definition of lead time in PSCNs should integrate with the supply chain network structure and operational details. Lead time corresponds to the response time in the worst case and assuming zero inventories. We define in this work the lead time of the PSCN as the length of the longest time path of chemical flows from a supplier to a customer by way of several manufacturing sites as shown in Figure 15, 16. The length of each time path is equal to the summation of all the time delays incurred by transportation and production in the path. If a plant or a transportation link is not selected, the associated delay is 0 . Binary variables are used to model the lead time $T P$ with the following inequalities:

$$
T P \geq Y_{k, l s}^{O} \lambda_{k, l s}^{O}+\sum_{x=1}^{n} Y_{k_{x}, i_{x}}^{P} \theta_{k_{x}, i_{x}}+\sum_{x=1}^{n-1} Y_{k_{x}, k_{x+1}}^{I} \lambda_{k_{x}, k_{x+1}}^{I}+Y_{k_{n}, l d}^{O} \lambda_{k_{n}, l d}^{O}
$$




$$
\forall\left(l s, k_{1}, k_{2} \ldots k_{n}, l d\right) \in \operatorname{Path}_{l s, k, l}
$$

According to constraint (31), the length of each time path of chemical flow is equal to the summation of products of all the corresponding binary variables (for installation of transportation links and manufacturing plants) multiplied by the associated time delay (by transportation or production). Furthermore, the lead time must be greater than the lengths for all the time paths. The set Path $_{l s, k, l}$ includes all the possible paths of chemical flow in a given potential PSCN network. All the elements in the set Path $_{l s, k, l}$ are in the form of $\left(l s, k_{1}, k_{2} \ldots k_{n}, l d\right)$, where supplier $l_{s}$ is the start of the path, $k_{1}, k_{2} \ldots k_{n}$ are the manufacturing sites that the associated chemical flow goes through, and customer $l d$ is the end of the path of the chemical flow.

It is obvious that the time delay in each transportation link is equal to the transportation time, and for dedicated process, the time delay by production is equal to the residence time.

The production time delay for a multiproduct plant is not so obvious. Before introducing our definition, consider a motivating example as shown in Figure 10 and 17. A multi-product plant produces three chemicals $A, B$ and $C$. Assume there is a demand change of chemical $A$. The worst case is when we just finished producing $A$, and there is no extra inventory of $A$ besides those committed to former demand. Then we must wait until the plant produces $A$ again, so that we can adjust the production to meet the demand change. This takes some time which is given by the processing time of chemical $B$ and $C$, plus residence time of $A$. In this way we define for multiproduct plant, the time delay for each product as cycle time plus residence time minus its processing time. Therefore, the time delay by production for a multiproduct plant is equal to the maximum time delay for each product: 


$$
\theta_{k, i} \geq T C_{k, i, t}+\theta_{i, s}^{R}-\sum_{s l \in S L_{i}} \delta_{k, i, s, s l, t} \quad \forall k \in K_{i}, i \in I_{j}
$$

In this definition, cycle times of each plant are taken into account as part of the delay due to production, so that we have integrated the production details into the quantitative definition of responsiveness.

The bi-linear terms (binary variable times the time delay of flexible process) in the lead time definition can be linearized. We use a continuous variable $T Y_{k, i}$ to replace the $Y_{k, i}^{P} \theta_{k, i}$ term in the lead time constraint:

$$
\begin{array}{r}
Y_{k, i}^{P} \theta_{k, i}=T Y_{k, i} \\
T P \geq Y_{k, l s}^{O} \lambda_{k, l s}^{O}+\sum_{x=1}^{n} T Y_{k_{x}, i_{x}}+\sum_{x=1}^{n-1} Y_{k_{x}, k_{x+1}}^{I} \lambda_{k_{x}, k_{x+1}}^{I}+Y_{k_{n}, l d}^{O} \lambda_{k_{n}, l d}^{O} \\
\forall\left(l s, k_{1}, k_{2} \ldots k_{n}, l d\right) \in \operatorname{Path}_{l s, k, l}
\end{array}
$$

The equation (33.1) is equivalent to the following disjunction:

$$
\left[\begin{array}{c}
Y_{k, i}^{P} \\
T Y_{k, i}=\theta_{k, i}
\end{array}\right] \vee\left[\begin{array}{c}
\neg Y_{k, i}^{P} \\
T Y_{k, i}=0
\end{array}\right]
$$

Applying the convex hull reformulation (Balas, 1985) to the above disjunctive constraint leads to:

$$
\begin{array}{ll}
T Y_{k, i}+T Y 2_{k, i}=\theta_{k, i} & \forall k, i \\
T Y_{k, i} \leq \theta_{k, i}^{U} Y_{k, i}^{P} & \forall k, i \\
T Y 2_{k, i} \leq \theta_{k, i}^{U}\left(1-Y_{k, i}^{P}\right) & \forall k, i
\end{array}
$$

where $T Y 2_{k, i}$ is a new continuous variable introduced as a slack variable.

It is interesting to note that this model reduces to a mixed-integer linear programming (MILP) problem if all the processes are dedicated, since the nonlinear 
terms in the lead time constraints are linearized and the time delays of the dedicated plants are known.

\subsection{Nonnegative Constraints}

All continuous variables must be nonnegative and the binary variables should be integer:

$$
\begin{aligned}
& Q_{k, i}, W_{k, i, j, s, t}, P_{k, j, l s, t}, T R_{k, k^{\prime}, j, t}, S_{k, j, l d, t}, T P \geq 0 \\
& Z_{k, i, s, s^{\prime}, s l, t}, W_{k, i, s, t}^{S}, r_{k, i, s}, \delta_{k, i, s, s l, t}, \delta_{k, i, s l, t}, T C_{k, i, t}, T e_{k, i, s l, t}, T s_{k, i, s l, t}, N_{k, i, t}, \theta_{k, i}, C O S T_{k, i, t}^{S} \geq 0 \\
& Y_{k, l}^{O}, Y_{k, i}^{P}, Y_{k, k^{\prime}}^{I}, Y_{m, l d}^{S}, Y_{k, i, s, s l, t}^{S} \in\{0,1\}
\end{aligned}
$$

\subsection{Net Present Value}

The NPV of the supply chain network is given by the following equations,

$$
\begin{aligned}
& N P V=\text { Income }-C_{\text {purchase }}-C_{\text {operate }}-C_{\text {tranport }}-C_{\text {invest }} \\
& \text { Income }=\sum_{k} \sum_{j} \sum_{l d} \sum_{t} \varphi_{j, l d, t} S_{k, j, l d, t} \\
& C_{\text {purchase }}=\sum_{k} \sum_{j} \sum_{l s} \sum_{t} \varphi_{j, l s, t} P_{k, j, l s, t} \\
& C_{\text {operate }}=\sum_{k} \sum_{i} \sum_{s} \sum_{j \in J P_{i, s}} \sum_{t} \sigma_{i, s, t} W_{k, i, j, s, t}+\sum_{k} \sum_{i} \sum_{t} \operatorname{COS} T_{k, i, t}^{S} \\
& C_{\text {tranport }}=\sum_{k} \sum_{j} \sum_{l s} \sum_{t} \omega_{k, j, l s, t}^{O} P_{k, j, l s, t}+\sum_{k} \sum_{k^{\prime}} \sum_{j} \sum_{t} \omega_{k, k^{\prime}, j, t}^{I} T R_{k, k^{\prime}, j, t}+\sum_{k} \sum_{j} \sum_{l d} \sum_{t} \omega_{k, j, l d, t}^{O} S_{k, j, l d, t} \\
& C_{\text {invest }}=\sum_{k} \sum_{i} \omega_{k, i}^{P} Q_{k, i}+\sum_{k} \sum_{i} \gamma_{k, i}^{P} Y_{k, i}^{P}+\sum_{k} \sum_{l} \gamma_{k, l}^{O} Y_{k, l}^{O}+\sum_{k} \sum_{k^{\prime}} \gamma_{k, k^{\prime}}^{I} Y_{k, k^{\prime}}^{I}
\end{aligned}
$$

All the parameters in the above formulation are discounted at a specified interest rate and include the effect of taxes on the net present value. 


\subsection{Solution Procedure}

In order to obtain the Pareto-optimal curve for a bi-criterion optimization problem, one of the objectives is specified as an inequality with a fixed value for the bound which is treated as a parameter. There are two major approaches to solve the problem in terms of this parameter. One is to simply solve it for a specified number of points to obtain an approximation of the Pareto optimal curve. The other is to solve the problem as a parametric programming problem (Dua and Pistikopoulos, 2004), which yields the exact solution for the Pareto optimal curve. While the latter provides a rigorous solution approach, the former one is simpler to implement. For this reason we have selected this approach. The procedure includes the following three steps: The first one is to minimize the lead time $T P$ to obtain the shortest lead time $T P_{S}$, which in turn yields the lowest Pareto optimal $N P V$. The second step is to maximize $N P V$ that in turn yields the longest Pareto optimal lead time $T P_{L}$. In this case the objective function is set as $N P V-\varepsilon \cdot T P$ ( $\varepsilon$ is a very small value, e.g., it is on the order of 0.001). The last step is to fix the lead time $T P$ to discrete values between $T P_{S}$ and $T P_{L}$, and optimize the model by maximizing $N P V$ at each selected point. In this way we can obtain an approximation to the Pareto-optimal curve, together with the optimal configurations of PSCN for different values of lead time.

\section{NUMERICAL EXAMPLE}

In this section we consider the PSCN for producing polystyrene resins in the motivating example (as shown in Figure 1). There are four suppliers, four customers, three manufacturing sites and three types of processes. Process $I$ is dedicated; Process $I I$ and III are flexible multiproduct plants. The lower bounds of the demands are set to zero, which implies that the model can select which customer markets to include in 
the optimal supply chain network. The time horizon is 10 years, and three time periods are considered, with length of 2 years, 3 years and 5 years, respectively. An annual interest rate of $5 \%$ has been considered for the calculation of the net present value.

The model consists of 133 binary variables, 2249 continuous variables and 3041 equations. It was modeled with GAMS (Brooke et al, 1998). Due to the non-convexity of the MINLP, it was solved using the BARON solver (Sahinidis, 1996) on an Intel 3.2 GHz machine with 512 MB memory. Nine points in the Pareto optimal curve take 133 hours computational time for global optimum.

The Pareto curve is shown in Figure 18, which required 133 hours of computation. From this figure, it can be seen that the Pareto curve ranges from 8.85 to 14.4 days in the lead time, and from \$159 million to \$1261 million for the NPV. It is interesting to see that in the range between 11.6 and 13.7 days, the NPV remains constant at \$794 million, which means that the lead time of 11.6 days can be obtained over a range with no penalty to the NPV.

The optimal network structures under different lead times are shown in Figure 19-22. It is interesting to see that all the three sites are selected, but different types of plants are installed in all the network structures. With the shortest lead time, 8.85 days, (Figure 19) only the customer markets in NY and GA are selected to sell the products, four plants in the three sites are installed and all the four suppliers are selected and connected to the associated nearest plant sites. As the lead time increases to 9.55 days, the customer market in MN can be linked to the AL manufacturing site, which leads to larger sales, in turn increasing the NPV (Figure 20). As shown in Figure 21, Plant $I$ in PA site and the links between suppliers to PA site are removed, while a new intersite transportation link from TX site to PA site for the transportation of styrene 
monomer is added. The change of network structure reduces the total investment cost, but also increase the lead time. With the longest lead time of 14.42 days, all the four customer markets are connected to the plant sites, and this structure leads to the highest NPV up to $\$ 1.2$ billon (Figure 22, 23). This example then shows the importance of establishing trade-offs between responsiveness and economics in the design and planning of a PSCN for the improvement of overall earning and performance of a company.

\section{CONCLUSIONS}

This chapter has presented a quantitative approach for designing responsive supply chain. Lead time under the assumption of zero inventories was proposed as a measure of responsiveness, and defined quantitatively with integration of supply chain network structure and operational details. A multiperiod mixed integer nonlinear programming (MINLP) mathematical model was developed to support the decision-making in design and planning of process supply chain networks. The model integrates the longterm strategic decisions (e.g. installation of plants, selection of suppliers, manufacturing sites and transportation links) with the short-term operational decisions (e.g. product changeovers and inventory profiles for flexible processes) for the multisite multi-echelon process supply chain network.

A bi-criterion optimization framework was implemented to obtain the trade-offs between responsiveness and economics in this model. The multi-objective optimization model was solved with the $\varepsilon$-constraint method for the sake of computational simplicity. An example reflected to styrene production was solved to illustrate the industrial application of this model. The result shows that small changes in lead time can lead to significant changes in the net present value and the network 
structure, which in turn suggests the importance of integrating responsiveness into the design and operations of process supply chain network.

In terms of the limitations of this model, we have assumed that all the transportation links are continuous and that there are no capacity expansions over the whole time horizon. Our next aim is to further develop this model so that it can accurately capture all the aspects of the PSCN, such as capacity expansion and the inventory effect of single product plants due to intermittent transportation. Uncertainties in the demands and prices of chemicals should also be taken into account. Finally, a more efficient algorithm to solve this type of nonconvex MINLP problem also needs to be developed.

\section{Acknowledgements}

The authors gratefully acknowledge financial support from National Science Foundation under Grant No. DMI-0556090. 


\section{Nomenclature}

\section{Indices/Sets}

$k \quad$ Manufacturing Sites

$i \quad$ Processes

$j \quad$ Chemicals

ls Suppliers

ld Customers

$l \quad$ Markets (suppliers and customers)

$t \quad$ Time periods

$s, s^{\prime} \quad$ Production schemes

$K_{i} \quad$ Set of sites that can set up process $i$

$J_{i, s} \quad$ Set of chemicals involved in scheme $s$ of process $i$

$J P_{i, s} \quad$ Set of main products for production scheme $s$ of process $i$

$J P_{k, i} \quad$ Set of main products of process $i$ in site $k$

$J M_{k, i} \quad$ Set of materials of process $i$ in site $k$

$S_{i} \quad$ Set of production schemes for process $i$

$S L_{i} \quad$ Set of time slot for process $i$ in the production scheduling

$L S_{j} \quad$ Set of suppliers that supply chemical $j$

$L D_{j} \quad$ Set of customers that need chemical $j$

$I_{j} \quad$ Set of processes that consume chemical $j$

$O_{j} \quad$ Set of processes that produce chemical $j$

Path $_{l s, k, l} \quad$ Set of possible path of chemical flow from a supplier to some sites and then ends at a customer. Elements are in the form of $\left(l s, k_{1}, k_{2} \ldots k_{n}, l d\right)$

\section{Parameters}

Lenp $_{t} \quad$ Length of each time period $t$

$d_{j, d, t}^{L} \quad$ Lower bound of demand of chemical $j$ in market $l d$ during time period $t$

$d_{j, l d, t}^{U} \quad$ Upper bound of demand of chemical $j$ in market $l d$ during time period $t$

$\varphi_{j, l d, t} \quad$ Price of chemical $j$ in market $l$ during time period $t$

$\varepsilon_{j} \quad$ Inventory cost of chemical $j$

$\theta_{i, s}^{R} \quad$ Residence time of the main product for production scheme $s$ of process $i$

$\gamma_{k, l}^{O} \quad$ Setup cost of pipelines from suppliers to plant sites or from plant sites to customers

$\gamma_{k, k^{\prime}}^{I} \quad$ Fixed cost of inter-plant site transportation

$\gamma_{k, i}^{P} \quad$ Fixed cost of installation of each plant

$\omega_{k, j, l, t}^{O} \quad$ Variable transportation cost of chemicals between markets $l$ and sites $k$

$\omega_{k, k^{\prime}, j, t}^{I}, \quad$ Unit transportation cost of chemicals for inter-plant site transportation 
$\omega_{k, i}^{P} \quad$ Variable cost of installation of each plant

$\lambda_{k, l}^{O} \quad$ Transportation time from between markets and plant sites

$\lambda_{k, k^{\prime}}^{I} \quad$ Transportation time from one plant site to the other

$\rho_{i, s} \quad$ Relative maximum production rate of main product in scheme s of process $i$

$\mu_{i, j, s} \quad$ Material balance coefficients of chemical $j$ in scheme $s$ of process $i$

$\sigma_{i, s, t} \quad$ Unit operating cost of scheme $s$ of process $i$ during period $t$

$\tau_{i, s, s^{\prime}} \quad$ Transition time from production scheme $S^{\prime}$ to $s$ in process $i$

$C T R_{i, s, s^{\prime}} \quad$ Transition cost from production scheme $s$ to $S^{\prime}$ in process $i$

$H_{k, i, t} \quad$ Total production time in plant $i$ of site $k$ in period $t$

$P_{k, j, l s, t}^{U} \quad$ Upper bound of purchase of chemical $j$ from supplier $l s$ to site $k$ during period $t$

$T R_{k, k^{\prime}, j, t}^{U} \quad$ Upper bound of transportation of product $j$ from site $k$ to $k^{\prime}$ in period $t$

$S_{k, j, l d, t}^{U} \quad$ Upper bound of sales of product $j$ to market $l d$ from site $k$ during period $t$

$W_{k, i, j, s, t}^{U} \quad$ Upper bound of production of chemical $j$ in plant $i$ of site $k$ in period $t$

$Q_{k, i}^{U} \quad$ Upper bound of capacity of each plant

\section{Continuous Variables:}

$Q_{k, i} \quad$ Capacity of each plant

$r_{k, i, s} \quad$ Processing rate of production scheme $s$ in plant $i$ of site $k$

$W_{k, i, j, s, t} \quad$ Amount of chemical $j$ produced in plant $i$ of site $k$ in period $t$

$P_{k, j, l s, t} \quad$ Purchase of chemical $j$ from supplier $l s$ to site $k$ during period $t$

$S_{k, j, l d, t} \quad$ Sales of product $j$ to market $l d$ from site $k$ during period $t$

$T R_{k, k^{\prime}, j, t} \quad$ Transportation of product $j$ from site $k$ to $k^{\prime}$ in period $t$

TP Total lead time of the whole supply chain network

$N P V \quad$ Net present value of the supply chain network

$\theta_{k, i} \quad$ Time delay by production of process $i$ in site $k$

$T s_{k, i, s l, t} \quad$ Starting time of slot $s l$ in process $i$ of site $k$ in period $t$

$T e_{k, i, s l, t} \quad$ End time of slot $s l$ in process $i$ of site $k$ in period $t$

$\delta_{k, i, s, s l, t} \quad$ Processing time of scheme s in slot $s l$ of process $i$ in site $k$

$\delta_{k, i, s l, t} \quad$ Processing time of the time slot $s l$ of process $i$ in site $k$

$T C_{k, i, t} \quad$ Cycle time of process $i$ in site $k$ in period $t$

$W_{k, i, s, t}^{S} \quad$ Amount produced of main product in scheme $s$ of process $i$ of site $k$ in period $t$

$N_{k, i, t} \quad$ Number of cycle in plant $i$ of site $k$ in period $t$

$\operatorname{COST}_{k, i, t}^{S} \quad$ Total cost for inventories and transitions of process $i$ in site $k$ in period $t$

\section{Binary Variables}

$Y_{k, i}^{P} \quad 1$ if plant $i$ in site $k$ is installed 
$Y_{k, l}^{O} \quad 1$ if transportation link from site $k$ to market $l$ is set up

$Y_{k, k^{\prime}}^{I} \quad 1$ if transportation link from site $k$ to $k^{\prime}$ is set up

$Y_{k, i, s, s l, t}^{S} \quad 1$ if the slot $s l$ is assigned to the production scheme $s$ in process $i$ of site $k$ in period $t$

$Z_{k, i, s, s^{\prime}, s l, t} \quad 1$ if production scheme $s$ is preceded by production scheme $s^{\prime}$ in time slot $s l$ of process $i$ in site $k$ in period $t$ 


\section{REFERENCES}

1) Balas E., "Disjunctive Programming and a Hierarchy of Relaxations for Discrete Continuous Optimization Problems”, SIAM Journal on Algebraic and Discrete Methods, 1985, 6(3), 466-486

2) Bok, J. K.; Grossmann, I. E.; Park, S., "Supply Chain Optimization in Continuous Flexible Process Networks", Industrial and Engineering Chemistry Research, 2000, 39, 1279-1290

3) Brooke, A.; Kendrick, D.; Meeraus, A.; Raman, R., “GAMS- A User’s Manual”, GAMS Development Corp., 1998

4) Chen, C. L.; Wang, B. W.; Lee, W. C., "Multiobjective Optimization for a Multienterprise Supply Chain Network", Industrial and Engineering Chemistry Research, 2003, 42, 1879-1889

5) Christopher, M; Towill, D., "An integrated model for the design of agile supply chains", International Journal of Physical Distribution \& Logistics Management, 2001, 31, 235246

6) Christopher, M., "The Agile Supply Chain Competing in Volatile Markets”, Industrial Marketing Management, 2000, 29, 37-44

7) Christopher, M., "Logistics and Supply Chain Management”, Publisher: Prentice Hall, 2005

8) Dua V.; Pistikopoulos, E.N., "Parametric Optimization in Process Systems Engineering: Theory and Algorithms”, Proceeding of Indian National Science Academy, 2004, 69A, 429-444

9) Fisher, M.L., "What is the right supply chain for your product?", Harvard Business Review, 1997, 75(2), 105-116

10) Forrester, J., “Industrial Dynamics”, MIT Press, and John Wiley \& Sons, Inc., New York 1961

11) Geoffrion, A. M.; Graves, G. W., "Multi-commodity Distribution System Design by Benders Decomposition”, Management Science, 1974, 20, 822-844

12) Grossmann I. E., "Enterprise-wide Optimization: A New Frontier in Process Systems Engineering”, AICHE Journal, 2005, 51, 1846-1857

13) Halshall-Whitney, H.; Thibault, J., "Multi-objective optimization for chemical processes and controller design: Approximating and classifying the Pareto domain", Computer and Chemical Engineering, 2006, 30, 1155-1168

14) Holweg, M., "The three dimensions of responsiveness", International Journal of Operations \& Production Management, 2005, 25, 603-622

15) Jackson, J. R.; Grossmann, I. E., "Temporal Decomposition Scheme for Nonlinear Multisite Production Planning and Distribution Models", Industrial and Engineering Chemistry Research, 2003, 42, 3045-3055

16) Kallrath, J., "Combined strategic and operational planning: An MILP success story in chemical industry”, OR Spectrum, 2002, 24, 315

17) Kok, A. G.; Graves, S. C., "Supply Chain Management: Design, Coordination and Operation, Handbooks in Operations Research and Management Science”, Publisher: Elsevier, 2003

18) Lee, H. L.; Padmanabhan, V.; Whang, S., "Information distortion in a supply chain: the bullwhip effect”, Management Science, 1997, 43(4), 546-558

19) Matson, J. B.; McFarlane, D. C., "Assessing the responsiveness of existing production operations”, International Journal of Operations \& Production Management, 1999, 19(8), 765-784

20) Norton, L. C.; Grossmann, I. E., "Strategic Planning Model for Complete Process Flexibility”, Industrial and Engineering Chemistry Research, 1994, 33, 69-76

21) Pinto, J. M.; Grossmann, I. E., "Optimal cyclic scheduling of multistage continuous multiproduct plants”, Computer and Chemical Engineering, 1994, 18, 797-816 
22) Raman, R.; Grossmann, I. E., "Symbolic integration of logic in mixed integer linear programming techniques for process synthesis", Computer and Chemical Engineering, 1993, 27, 909

23) Sabath, R., "Volatile demand calls for quick response”, International Journal of Physical Distribution \& Logistics Management, 1998, 28(9/10), 698-704

24) Sahinidis, N. V., "BARON: A general purpose global optimization software package", Journal of Global Optimization, 1996, 8(2), 201-205

25) Schulz, E. P.; Diaz, M. S.; Bandoni, J. A., "Supply chain optimization of large-scale continuous processes", Computer and Chemical Engineering, 2005, 29, 1305-1316

26) Shah, N., "Process industry supply chains: Advances and challenges", Computer and Chemical Engineering, 2005, 29, 1225-1235

27) Shaw, N. E.; Burgess, T. F.; Mattos, C.; Stec, L. Z., "Supply chain agility: the influence of industry culture on asset capabilities within capital intensive industries", International Journal of Production Research, 2005, 43(16), 3497-3516

28) Sousa, R. T.; Shah, N.; Papageorgiou, L. G., "Supply Chain Design and Planning with Responsiveness Testing - a Two-level Holistic Approach to an Industrial Case”, AICHE Annual Meeting, San Francisco, CA, 2006

29) Stalk, G., "Time - The next Source of Competitive Advantage", Harvard Business Review, 1988, 66 (4): 41-51

30) Tsiakis, P.; Shah, N.; Pantelides, C. C., "Design of Multi-echelon Supply Chain Networks under Demand Uncertainty", Industrial and Engineering Chemistry Research, 2001, 40, 3585-3604

31) Wolsey, L. A., "MIP modeling of changeovers in production planning and scheduling problems”, European Journal of Operational Research, 1997, 99, 154-165

32) Yusuf, Y. Y.; Gunasekaran, A.; Adeleye, E. O.; Sivayoganathan, K., "Agile supply chain capabilities: Determinants of competitive objectives”, European Journal of Operational Research, 2004, 159, 379-392 


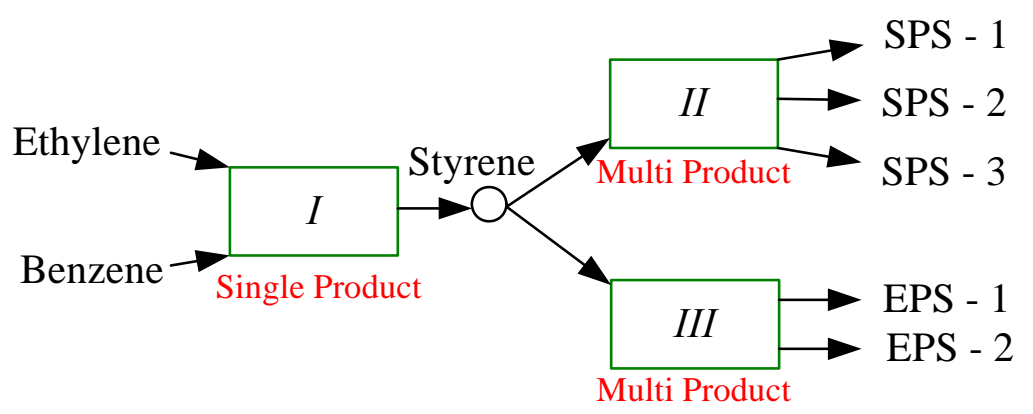

Figure 1. Production network for motivating example

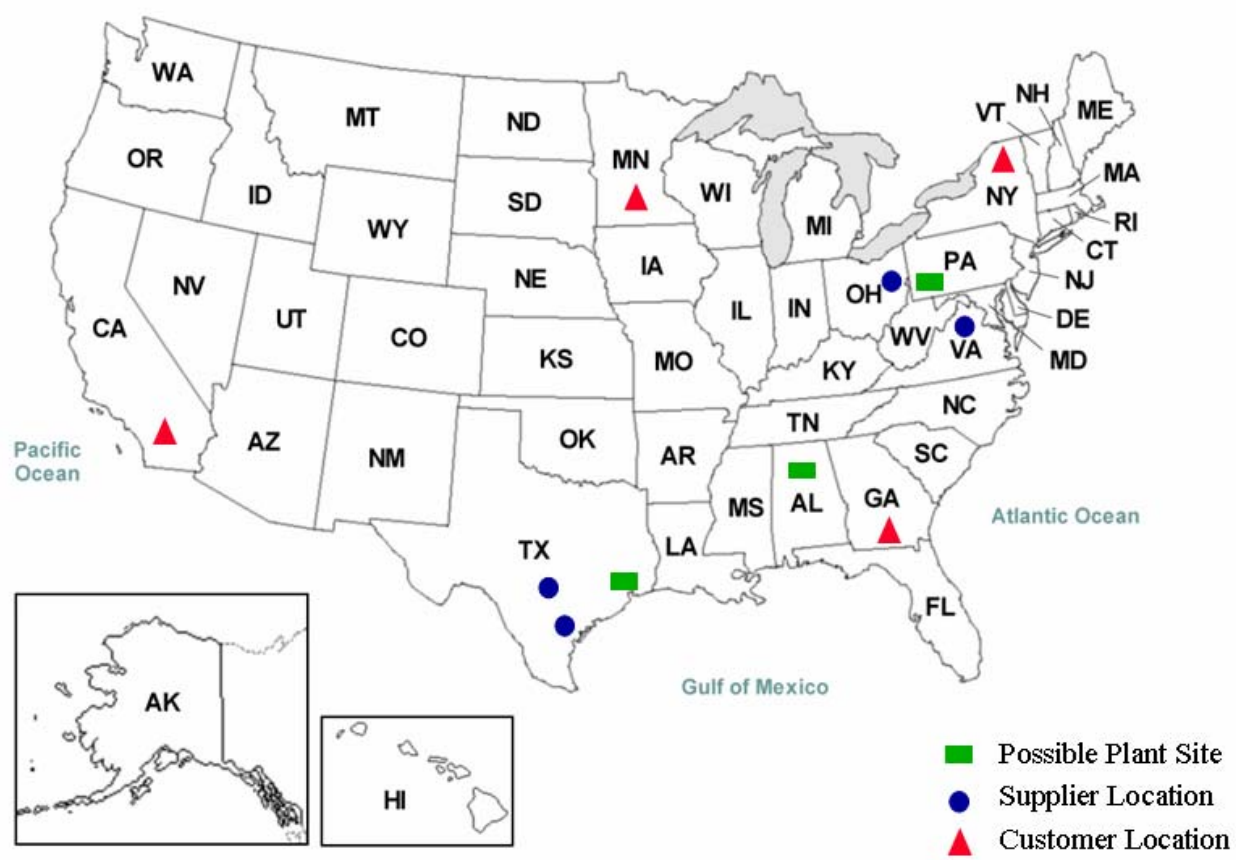

Figure 2. Location map for motivating example

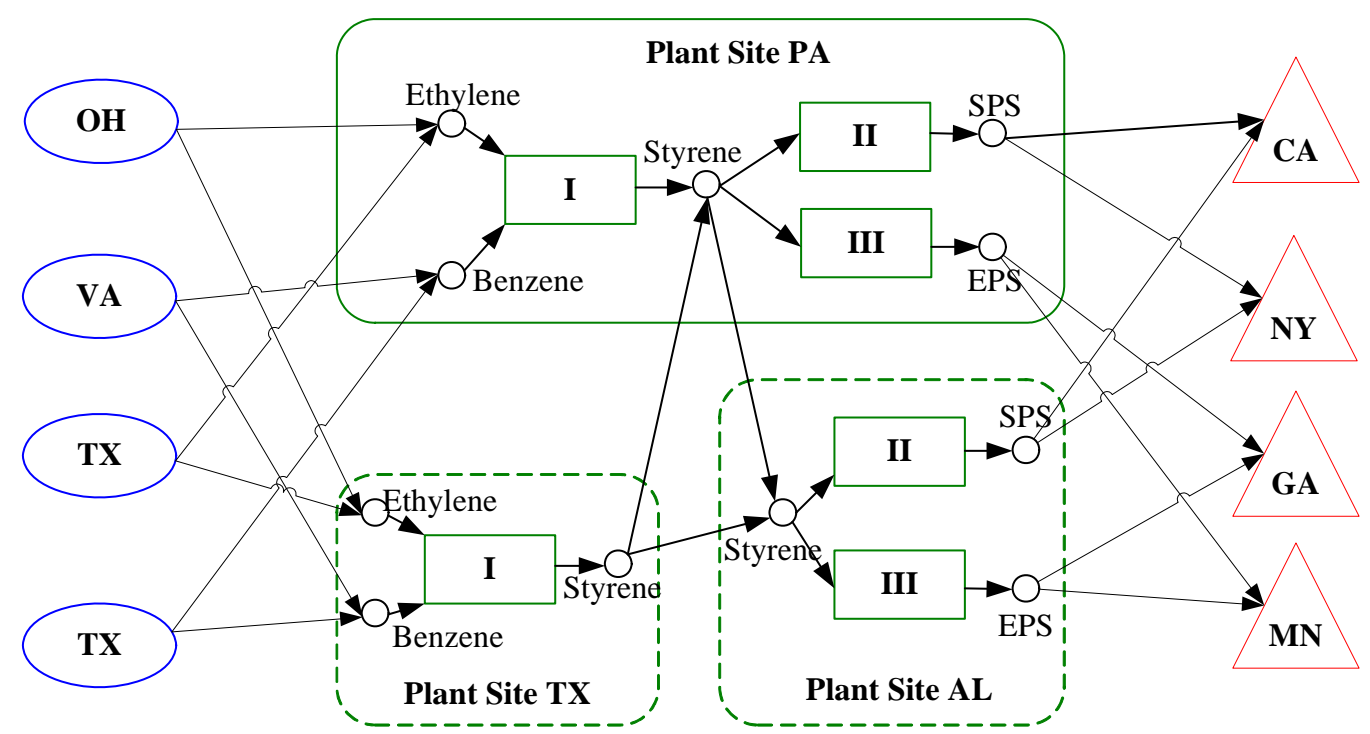

Figure 3. Supply chain network superstructure 

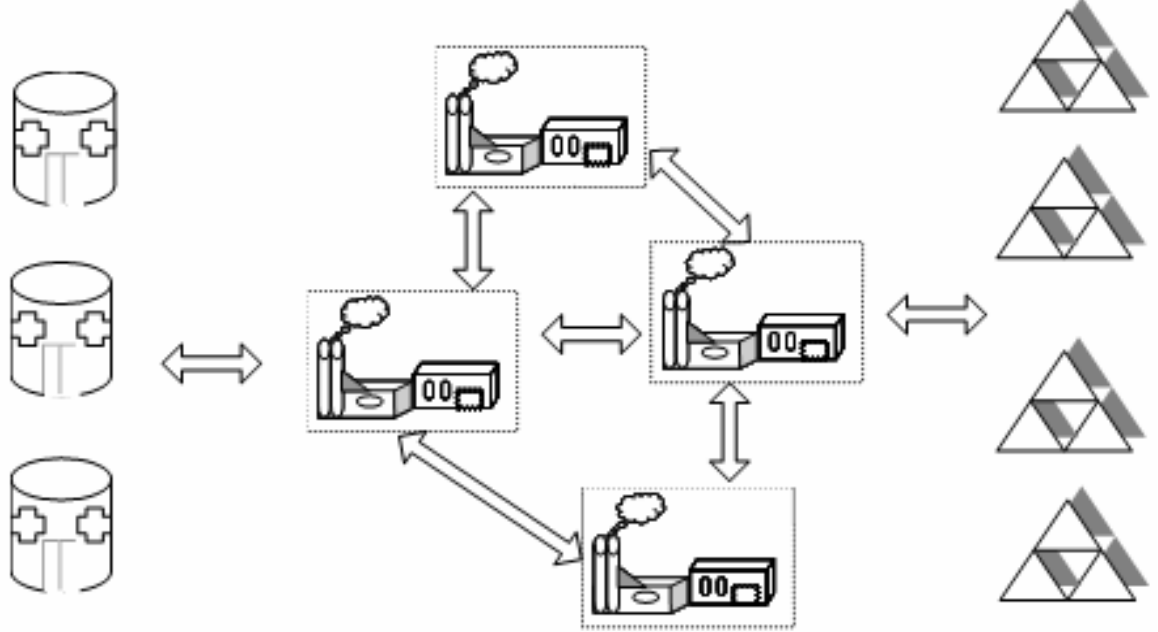

Suppliers

Production/Warehousing Node

\section{Customers}

Figure 4. Typical process supply chain network

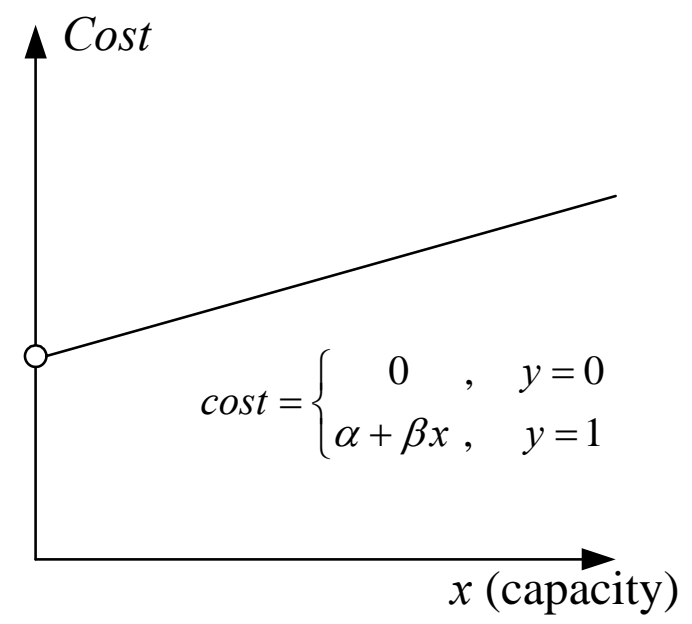

Figure 5. Cost function with fixed charge

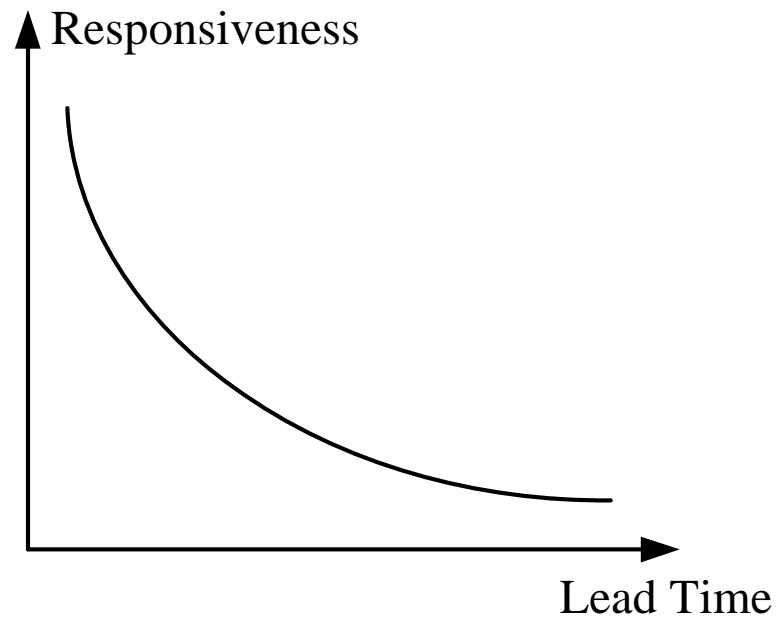

Figure 6. Conceptual relationship between lead time and responsiveness 


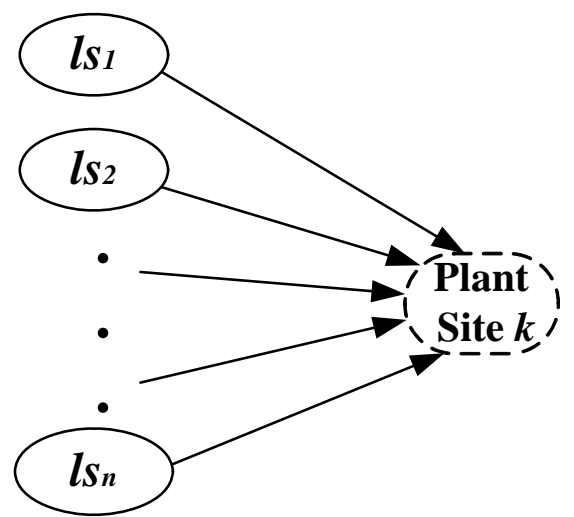

Figure 7. Relationship between suppliers and manufacturing sites

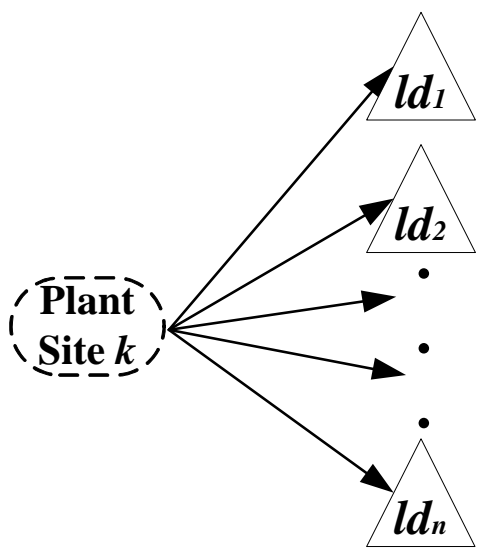

Figure 8. Relationship between manufacturing sites and customers

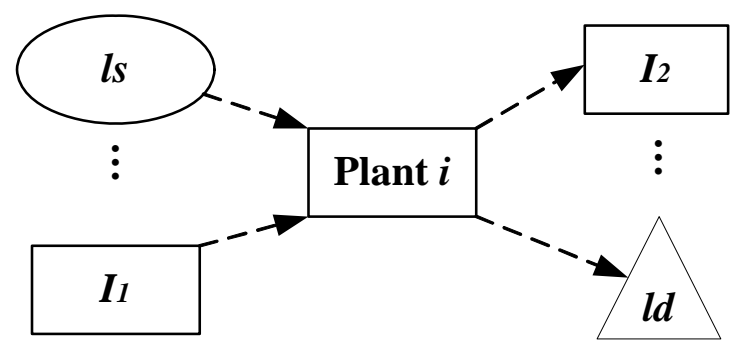

Figure 9. Input and output relationship of a plant
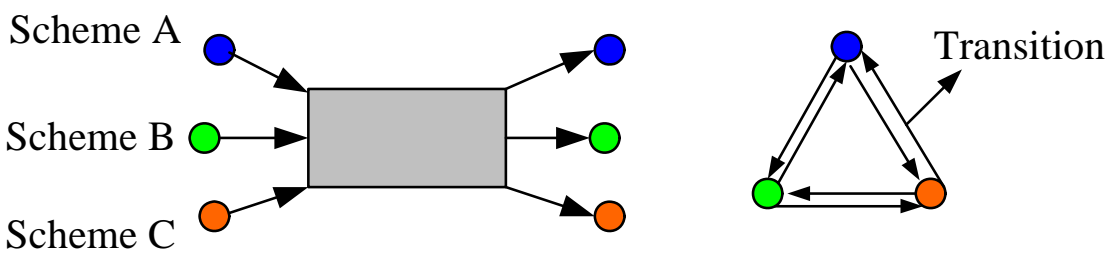

Figure 10. Changeovers of flexible processes 


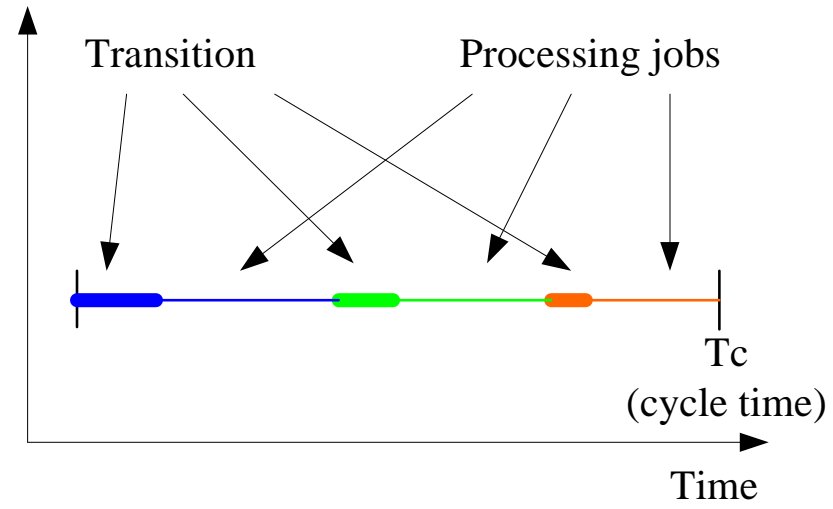

Figure 11. Cyclic scheduling in a cycle

The Whole Time Period

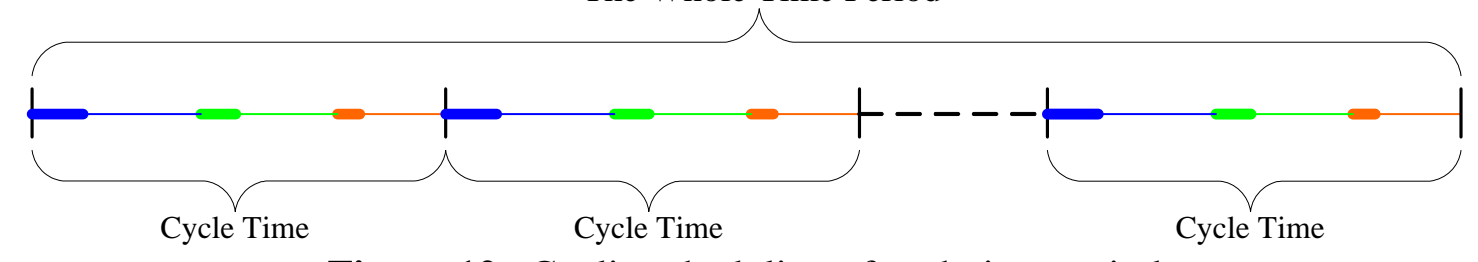

Figure 12. Cyclic scheduling of each time period

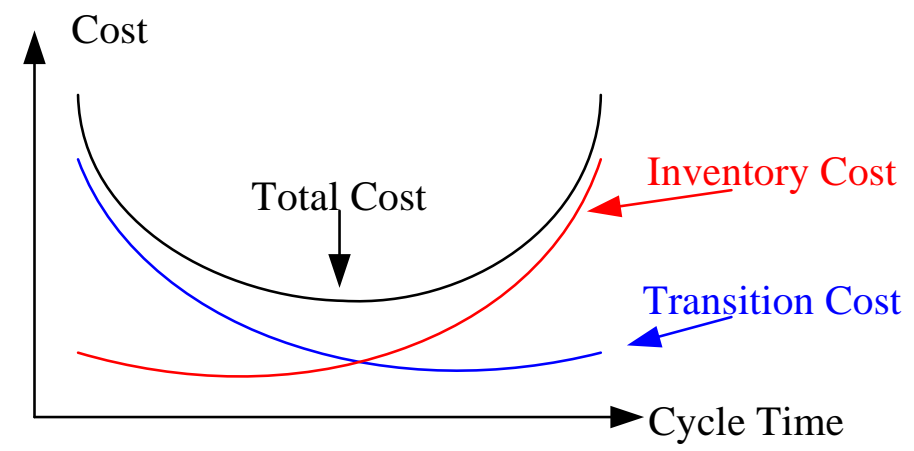

Figure 13. Trade-off between inventory and transition in cyclic scheduling

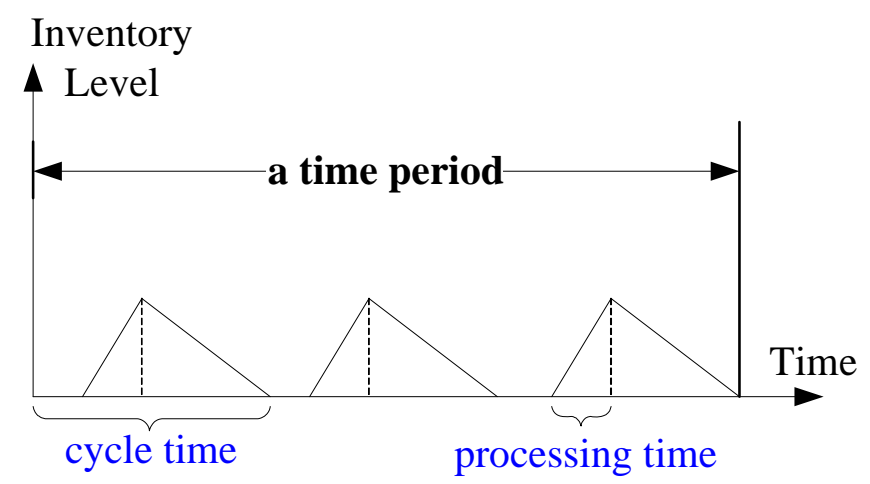

Figure 14. Inventory level change in cyclic scheduling 


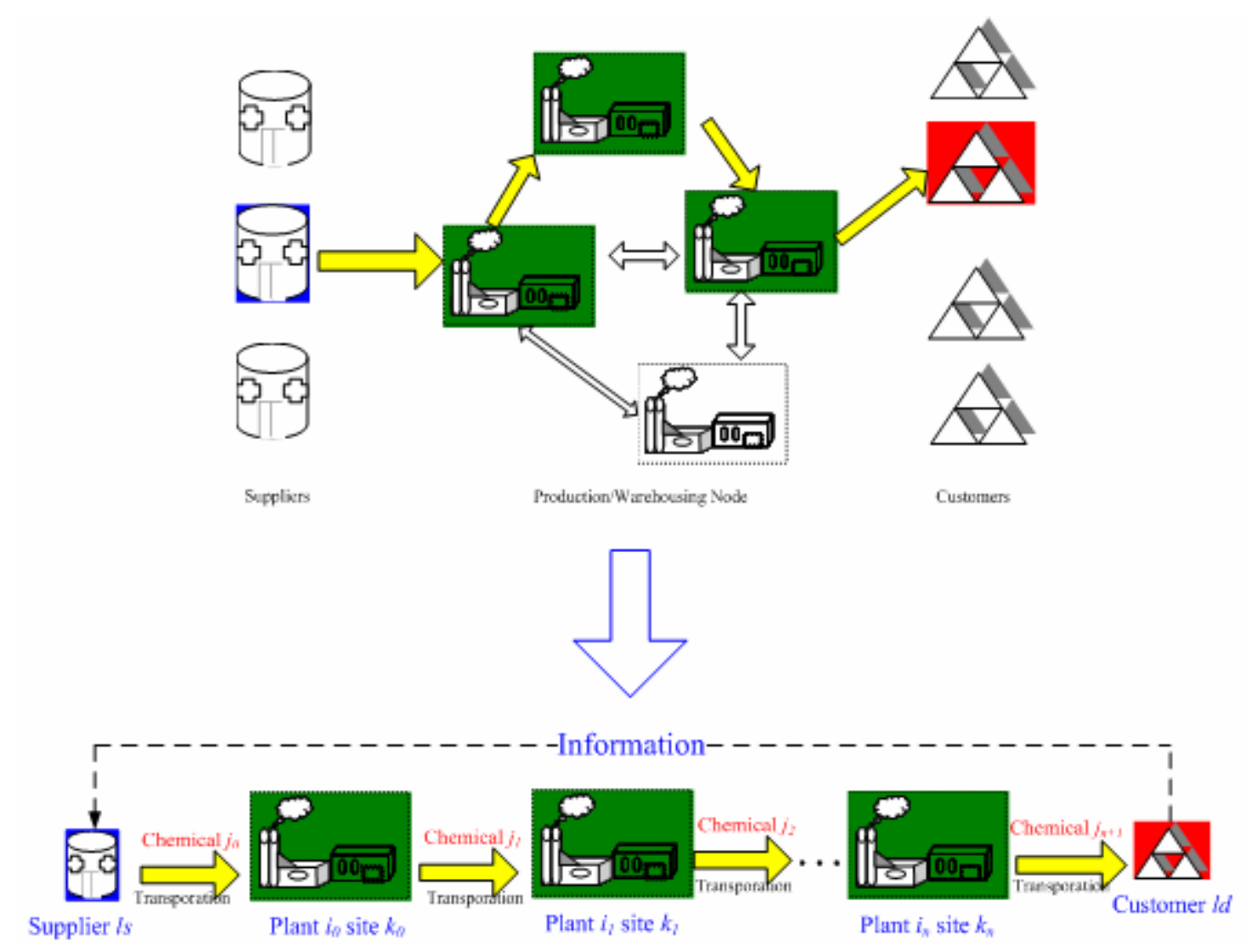

Figure 15. A path of chemical flow in a PSCN

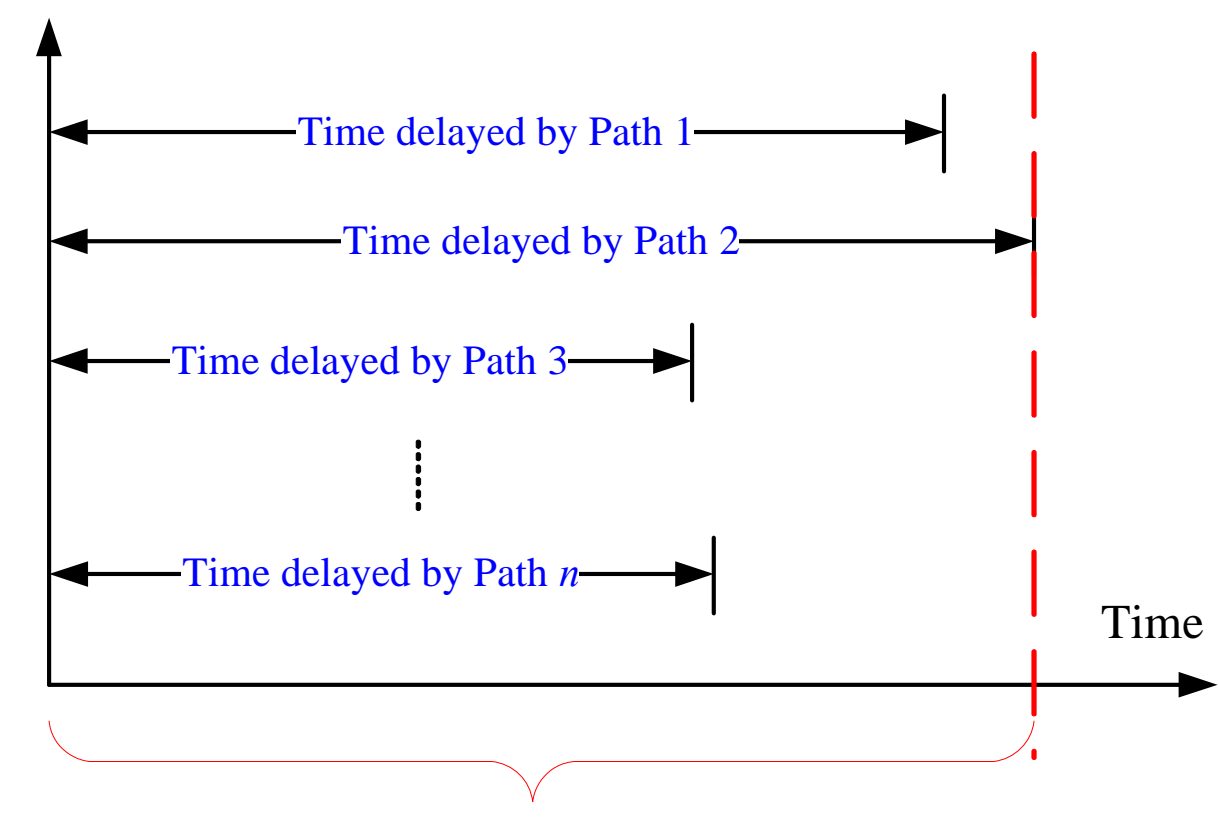

Lead Time

Figure 16. Lead Time of PSCNs 


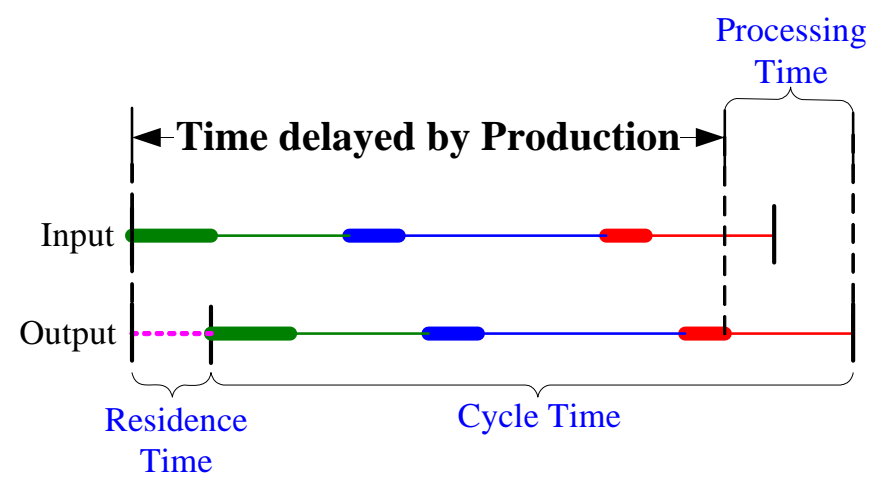

Figure 17. Time delay by production

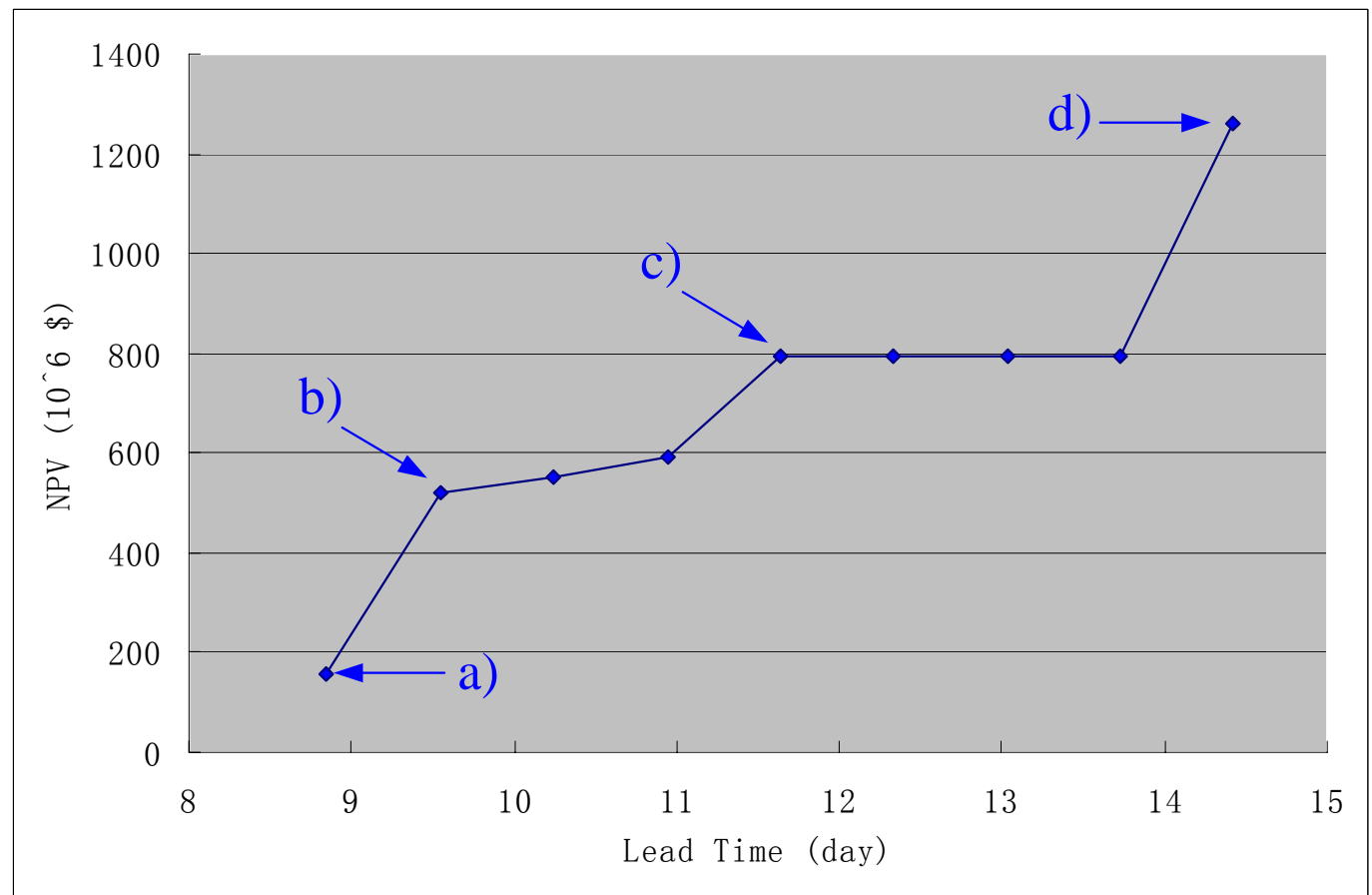

Figure 18. Pareto optimal curve for the numerical example

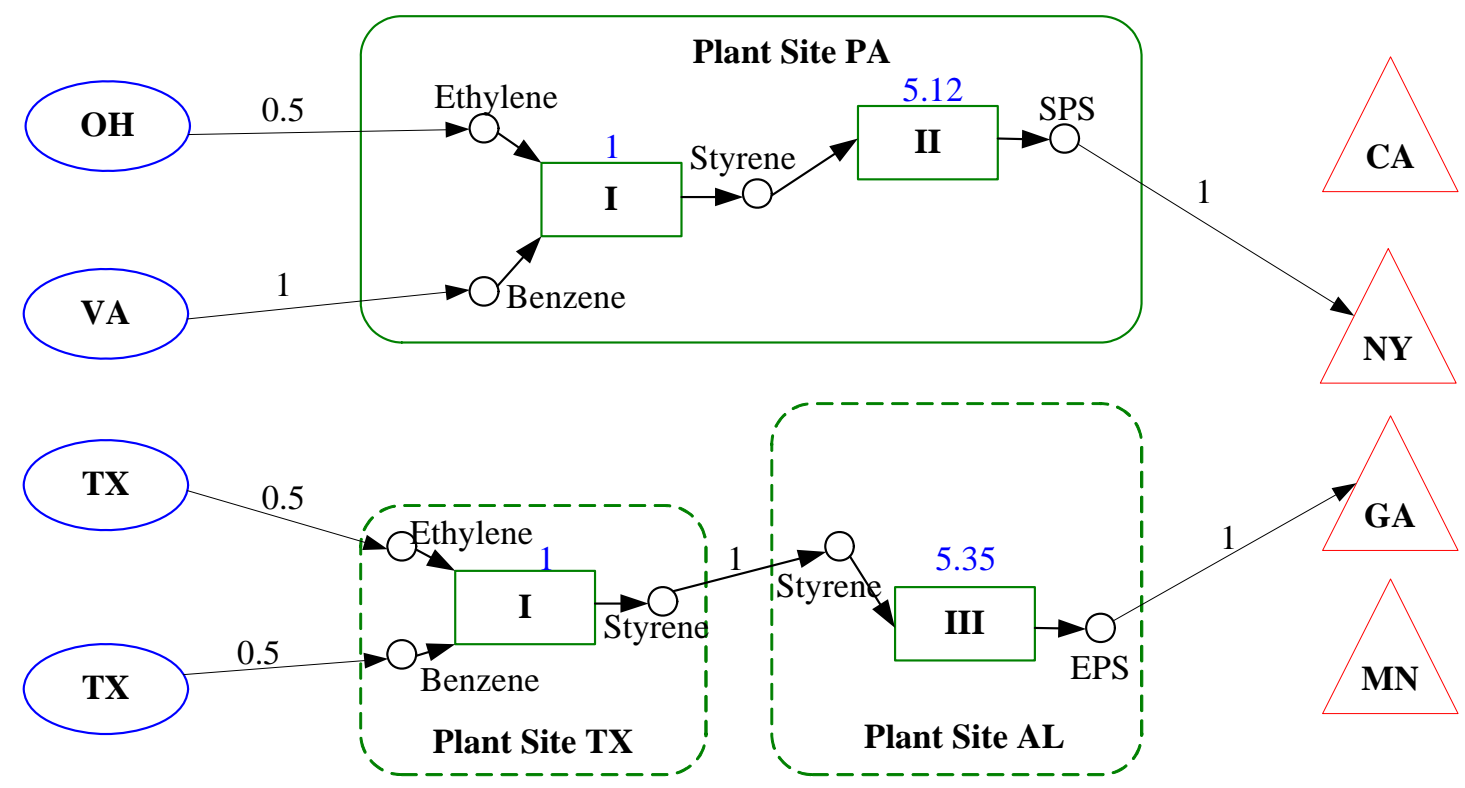

Figure 19. Optimal network structure at (a) lead time $=8.85$ days 


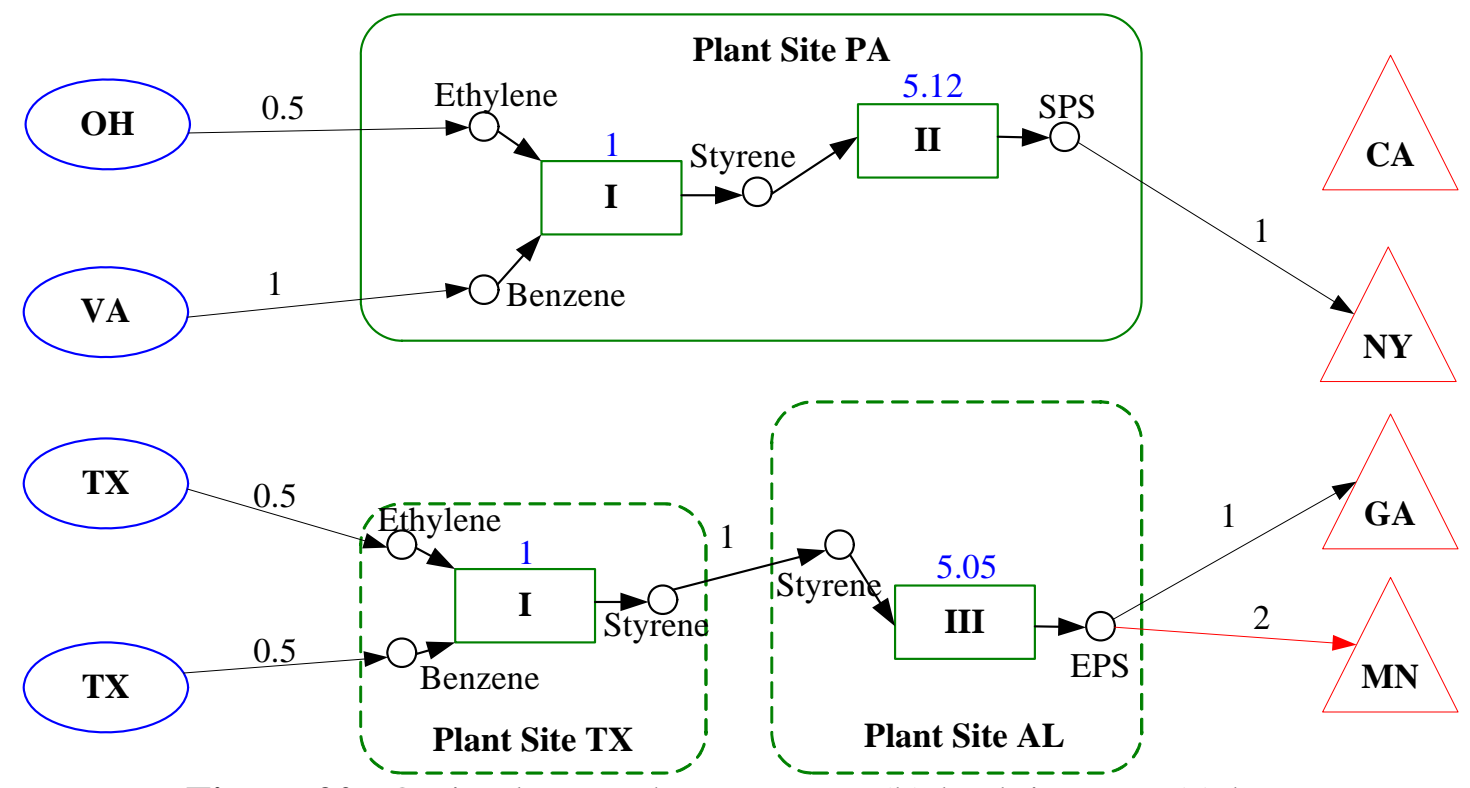

Figure 20. Optimal network structure at (b) lead time $=9.55$ days

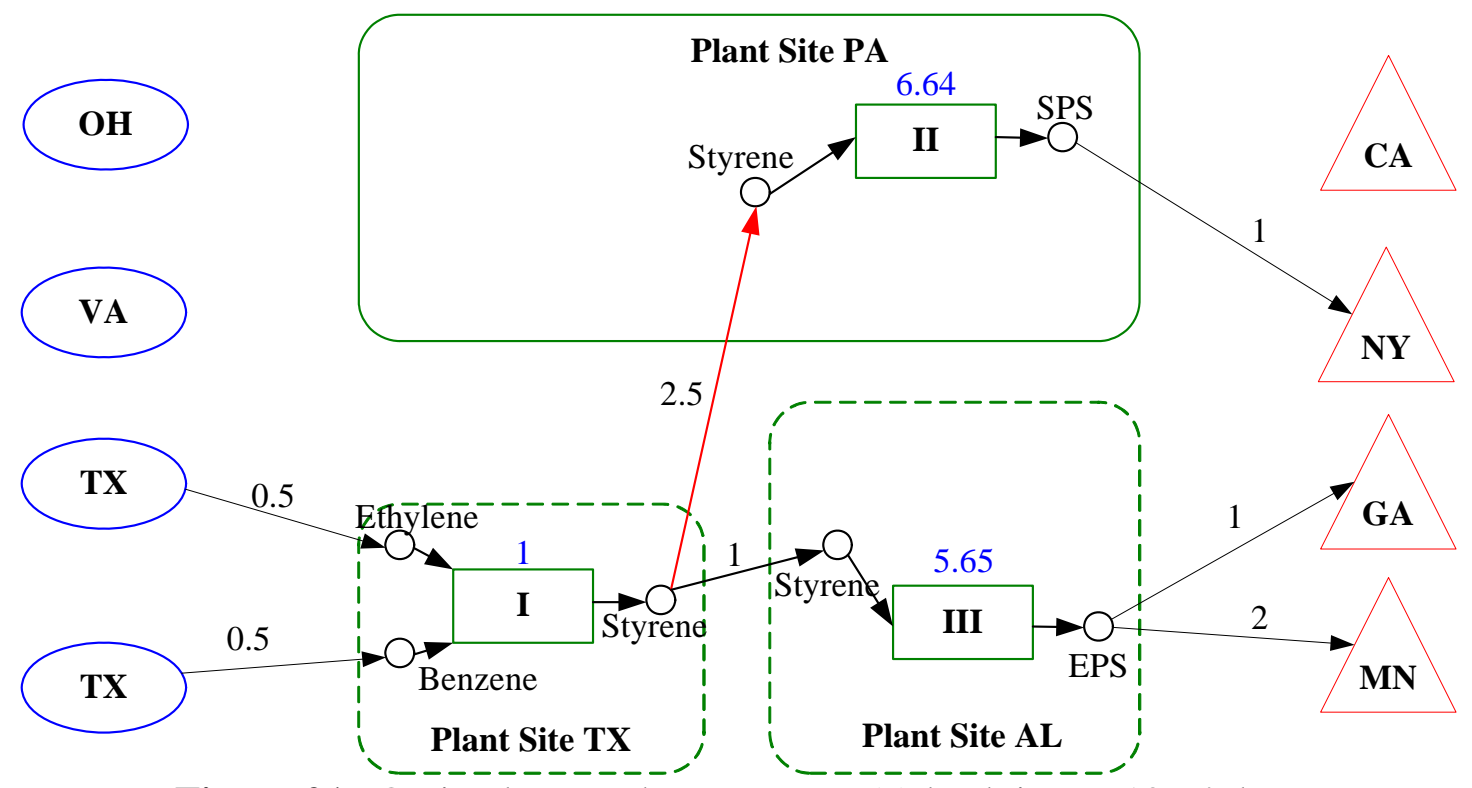

Figure 21. Optimal network structure at (c) lead time $=10.94$ days 


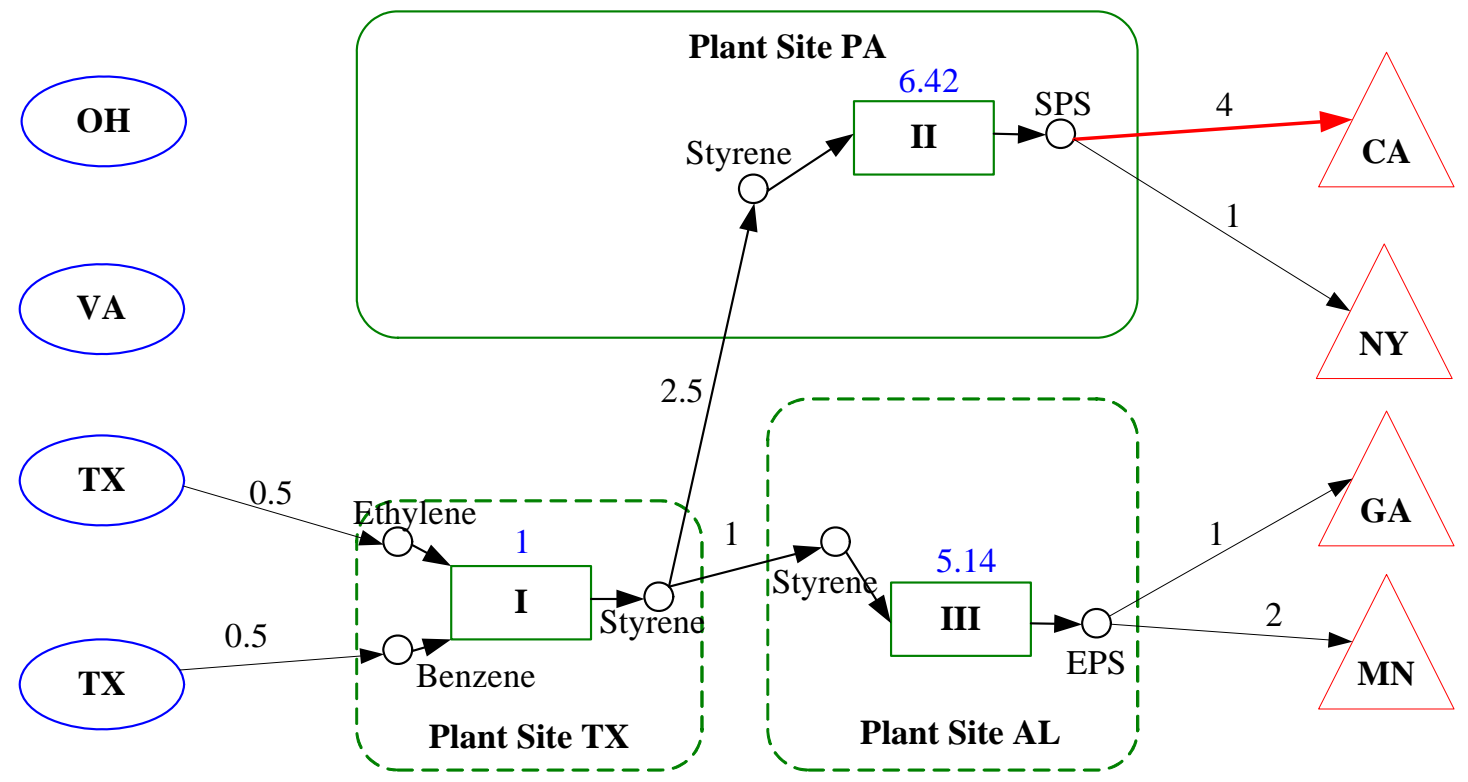

Figure 22. Optimal network structure at (d) lead time $=14.42$ days

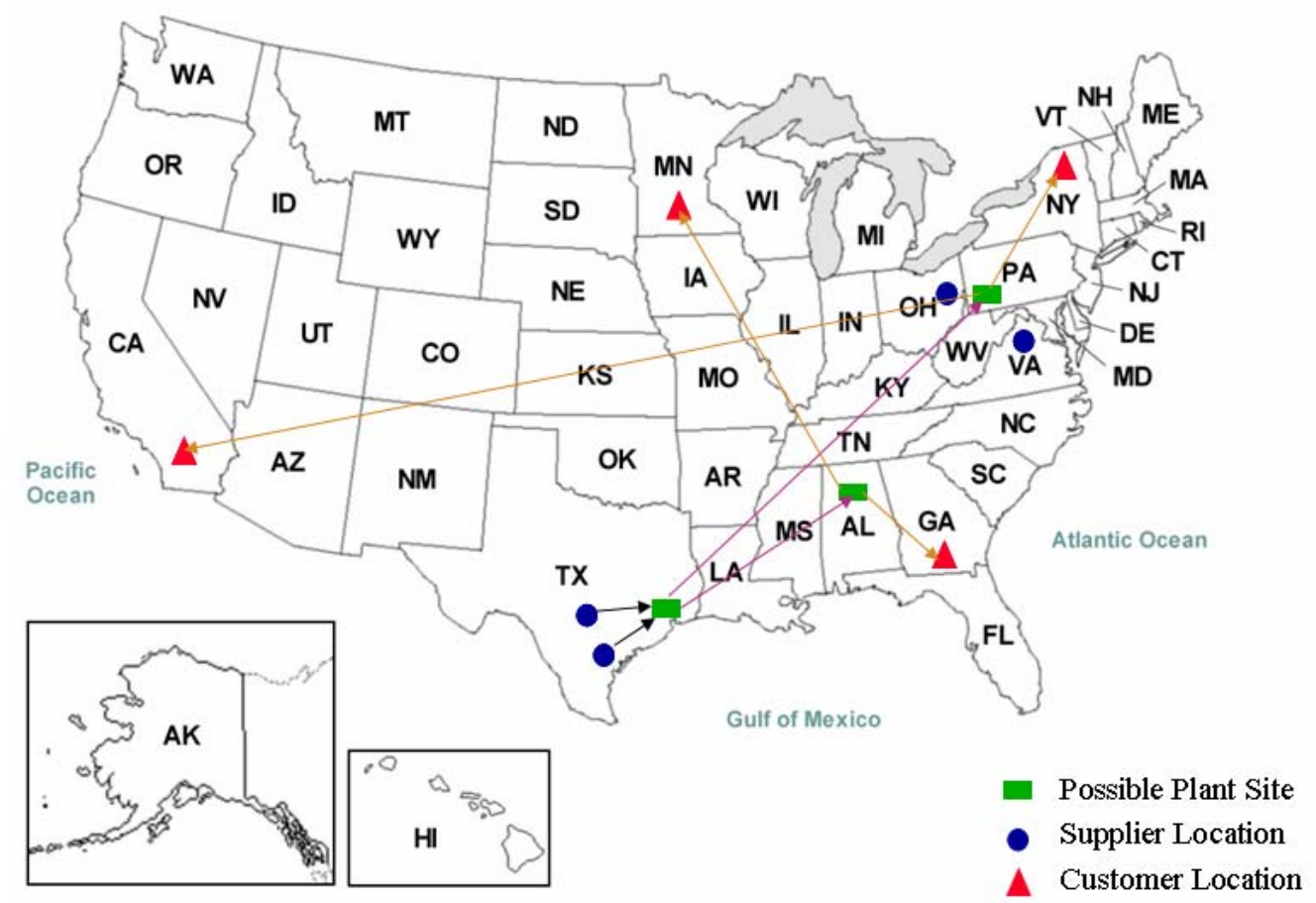

Figure 23. Material flows in the location map for longest lead time (14.42 days) case 